\title{
A PRESSURE-ROBUST DISCRETIZATION OF OSEEN'S EQUATION USING STABILIZATION IN THE VORTICITY EQUATION*
}

\author{
NAVEED AHMED ${ }^{\dagger}$, GABRIEL R. BARRENECHEA ${ }^{\ddagger}$, ERIK BURMAN§, JOHNNY \\ GUZMÁN 『, ALEXANDER LINKE", AND CHRISTIAN MERDON"
}

\begin{abstract}
Discretization of Navier-Stokes equations using pressure-robust finite element methods is considered for the high Reynolds number regime. To counter oscillations due to dominating convection we add a stabilization based on a bulk term in the form of a residual-based least squares stabilization of the vorticity equation supplemented by a penalty term on (certain components of) the gradient jump over the elements faces. Since the stabilization is based on the vorticity equation, it is independent of the pressure gradients, which makes it pressure-robust. Thus, we prove pressureindependent error estimates in the linearized case, known as Oseen's problem. In fact, we prove an $O\left(h^{k+\frac{1}{2}}\right)$ error estimate in the $L^{2}$-norm that is known to be the best that can be expected for this type of problem. Numerical examples are provided that, in addition to confirming the theoretical results, show that the present method compares favorably to the classical residual-based streamline upwind Petrov-Galerkin stabilization.
\end{abstract}

Key words. incompressible Navier-Stokes equations, divergence-free mixed finite element methods, pressure-robustness, convection stabilization, Galerkin least squares, vorticity equation

AMS subject classifications. $65 \mathrm{~N} 30,65 \mathrm{~N} 12,76 \mathrm{D} 07$

DOI. $10.1137 / 20 \mathrm{M} 1351230$

1. Introduction. In recent years, it has been observed that the saddle point structure of the incompressible Navier-Stokes equations

$$
\begin{aligned}
\boldsymbol{u}_{t}-\mu \Delta \boldsymbol{u}+(\boldsymbol{u} \cdot \nabla) \boldsymbol{u}+\nabla p & =\boldsymbol{f}, \\
\operatorname{div} \boldsymbol{u} & =0,
\end{aligned}
$$

induces, besides the fulfillment of the well-known discrete Ladyzhenskaya-BabuškaBrezzi (LBB) condition [8, 34], a second fundamental challenge [42]. This second challenge, which is closely related to the classical grad-div stabilization [31], is briefly described as follows: since the pressure acts as a Lagrangian multiplier for the divergence constraint, the pressure gradient $\nabla p$ will always balance any occurring, unbalanced gradient field in the momentum balance. Thus, gradient fields in the momentum balance do only change $\nabla p$, but not the velocity $\boldsymbol{u}$, which leads to the existence of certain equivalence classes of forces - and a corresponding seminorm - that determine

${ }^{*}$ Received by the editors July 8, 2020; accepted for publication (in revised form) June 14, 2021; published electronically October 28, 2021.

https://doi.org/10.1137/20M1351230

Funding: The work of the first author has been partially funded by Kuwait Foundation for the Advancement of Sciences (KFAS) (funding code is CN20-46SM-01). The work of the second author has been partially funded by the Leverhulme Trust through the Research Fellowship RF-2019-510.

${ }^{\dagger}$ Department of Mathematics and Natural Sciences, Gulf University for Science and Technology, Mubarak Al-Abdullah Area/West Mishref, Kuwait (ahmed.n@gust.edu.kw).

${ }^{\ddagger}$ Department of Mathematics and Statistics, University of Strathclyde, 26 Richmond Street, Glasgow, G1 1XH United Kingdom (gabriel.barrenechea@strath.ac.uk).

$\S$ Department of Mathematics, University College London, London, WC1E 6BT, United Kingdom (e.burman@ucl.ac.uk).

『Division of Applied Mathematics, Brown University, Providence, RI 02912 USA (johnny_ guzman@brown.edu).

"Weierstrass Institute for Applied Analysis and Stochastics (WIAS), Mohrenstr. 39, 10117 Berlin, Germany (linke@wias-berlin.de, merdon@wias-berlin.de).

$$
2746
$$


the solution structure of the problem [33]. The purpose of this work is to investigate the relation of this second challenge to the question of how to stabilize dominant advection in high Reynolds number flows.

Space discretizations that remain accurate in the presence of dominant gradient fields in the momentum balance - leading to strong pressure gradients - have recently triggered notable research activity $[35,55,46,44,28,61,20,39,40,2,45,56,57,47$, $25,26]$ and have been called pressure-robust $[48,3]$. We remark that the classical graddiv stabilization is a means to enhance the pressure-robustness of popular methods like the Taylor-Hood element [18]. The concept of pressure-robustness explains how these equivalence classes of forces and the special role of gradient-type forces affect the notion of dominant advection in Navier-Stokes flows. Starting from the idea of pressure-robustness, in this work we propose a novel discrete stabilization operator for Navier-Stokes flows that uses only the vorticity equation, and not the entire momentum equation. As a model we consider the linear steady-state Oseen equation discretized by means of an inf-sup stable pair of spaces using $H^{1}$-conforming velocities of polynomials of order $k$ and pressures of order $k-1$. To this discrete system, we add a Galerkin least squares-type term to the formulation involving the vorticity equation. One of the main results emerging from the analysis of the method is that we are able to prove the following error estimate in the convection dominated regime:

$$
\left\|\boldsymbol{u}-\boldsymbol{u}_{h}\right\|_{L^{2}} \leq C h^{k+\frac{1}{2}}|\boldsymbol{u}|_{H^{k+1}} .
$$

To the best of our knowledge, this closes a disturbing gap in the theory of mixed finite elements that had not been overcome yet. In fact, when $H^{1}$-conforming velocities are used, the same convergence order for the velocity error in the $L^{2}$ norm had been achieved for equal order interpolation methods only (see, e.g., [30, 10, 15, 11, 23]) or by adding bubble functions to the finite element space as in [51] - though at the price of an additional dependency of the velocity error on the continuous pressure $p$, i.e., giving up pressure-robustness. We remark further that enhancing popular methods like the Taylor-Hood element by a combined enhancement with grad-div stabilization and a standard convection stabilization does not lead to the optimal convergence rate (1.2) either [41], and the same pressure-dependency of the error estimate would occur if pressure stabilized methods, such as the ones given in $[12,7,14]$, were to be used. This demonstrates that convection stabilization of Oseen's problem does not merely require pressure-robustness and convection stabilization, but calls for a pressure-robust convection stabilization. Interestingly, whenever the degree of spaces is different (such as in the present case) only in the very recent paper [6] has such a pressure-robust estimate been proven for an incompressible flow problem, at the cost of giving up $H^{1}$-conformity. In fact, the spaces used in [6] were only $H$ (div)-conforming.

The main reason why an estimate such as (1.2) has not been obtained using infsup stable elements supplied with classical stabilization mechanisms is linked to the pressure gradient. In fact, when streamline upwind Petrov-Galerkin (SUPG) stabilization is used, the pressure must be included in the stabilizing terms for consistency, and the approximation of the pressure, being of a lower order than the one for velocity, prevents proving (1.2). Symmetric stabilization methods, such as continuous interior penalty, local projection stabilization, and the orthogonal subscales method have been successfully used for scalar convection-diffusion equations. When one of these methods is applied to the Oseen equation with inf-sup stable elements, the stabilization is independent of the pressure. So, in principle, the application of the same analysis 
from the convection-diffusion equation to the Oseen equation seems achievable. Nevertheless, a more detailed inspection shows that their stability and convergence rely on orthogonality properties of some interpolant and stabilization of the orthogonal complement of the convective term. Consistency is obtained since this orthogonal complement tends to zero at an optimal rate under refinement. Nevertheless, when pressure-robust spaces are used, similar orthogonality cannot be exploited for the vector-valued Oseen's problem since the convection term itself is not divergence-free in general [17]. So, the extension of the existing analysis for a scalar convectiondiffusion equation cannot be carried out unless the pressure gradients are eliminated. Based on this observation, in this work we add a stabilizing term that penalizes the equation for the vorticity, where pressure gradients are naturally absent, and no extra properties of the convective term are required. Since the equation to be penalized is the curl of the momentum equation, this is a stabilization linked to the so-called Galerkin gradient least squares stabilized methods (see, e.g., [29, 60]).

The structure of the manuscript is as follows. The introduction is completed by two short sections, one regarding the motivation and background for the new method introduced in this work, and one containing preliminary results about vector potentials for divergence-free functions and their regularity. Then, in section 2 we introduce the finite element method used in this work, along with various examples of finite element spaces that are appropriate for its use. In section 3, we deliver a detailed numerical analysis. We achieve optimal convergence orders for the discrete velocity in the $H^{(\Omega)^{d}}$ norm and, as stated earlier, the first $O\left(h^{k+\frac{1}{2}}\right)$ error estimate for the velocity in the $L^{2}$-norm for the convection-dominated regime. A supercloseness result for the discrete pressure, typical for pressure-robust discretizations, is also derived. In section 4, we will provide and discuss the results of testing the present method for different benchmark problems. The benchmarks cover both extreme cases, where the convection term is a gradient field or a divergence-free vector field. Also, the general case is treated, where the convection term is a sum of a gradient field and a divergence-free field. The new least squares vorticity stabilization is compared to a Galerkin discretization and a SUPG stabilization applied to the same pairs of finite element methods. The numerical results show the improvement provided by the new stabilized method over both the Galerkin and SUPG methods. Finally, some conclusions are drawn in section 5 .

1.1. Background, motivation, and notation. We start by setting the notation to be used throughout. We will use standard notation for Lebesgue and Sobolev spaces, in line with, e.g., [34]. In particular, for a domain $D \subseteq \mathbb{R}^{d}, d=2,3$, and $q \in[1,+\infty], L^{q}(D)$ will denote the space of measurable functions such that its $q$ th power is integrable in $D$ (for $q<+\infty$ ) and essentially bounded in $D$ (when $q=+\infty$ ). The space $L_{0}^{q}(D)$ denotes the space of functions in $L^{q}(D)$ with zero mean value in $D$. Its norm will be denoted by $\|\cdot\|_{0, q, D}$ (except when $q=2$, in which case we denote the norm by $\left.\|\cdot\|_{0, D}\right)$. In addition, the inner product in $L^{2}(D)$ will be denoted by $(\cdot, \cdot)_{D}$. For $k \geq 0$ the space $W^{k, q}(D)$ denotes all generalized functions that belong to $L^{q}(D)$ with distributional derivatives up to the $k$ th order belonging to $L^{q}(D)$. We will denote its norm (seminorm) by $\|\cdot\|_{k, q, D}\left(|\cdot|_{k, q, D}\right)$. When $q=2, W^{k, 2}(D)=H^{k}(D)$, and its norm (seminorm) is denoted by $\|\cdot\|_{k, D}\left(|\cdot|_{k, D}\right)$. The space $W_{0}^{k, q}(D)\left(H_{0}^{k}(D)\right)$ denotes the closure of $C_{0}^{\infty}(D)$ in $W^{k, q}(D)\left(H^{k}(D)\right)$. The space $H^{-1}(D)$ denotes the dual of $H_{0}^{1}(D)$ with respect to the inner product in $L^{2}(D)$, the corresponding duality pairing will be denoted by $\langle\cdot, \cdot\rangle_{D}$, and the associated norm is denoted by $\|\cdot\|_{-1, D}$. The vector-valued counterpart of a space $X$ will be denoted simply by $X^{d}$, and the same notation will be used for inner products, norms, and duality pairing. 
In order to motivate our new stabilization approach, we now reflect on the notion of dominant advection for the incompressible Navier-Stokes equations. We set the problem in a bounded, polyhedral, contractible domain $\Omega \subseteq \mathbb{R}^{d}, d=2,3$, with Lipschitz continuous boundary $\partial \Omega$. In addition, we define the space of divergence-free functions in $\Omega$ as

$$
\mathcal{V}(\Omega):=\left\{\boldsymbol{v} \in H_{0}^{1}(\Omega)^{d} \text { such that } \operatorname{div} \boldsymbol{v}=0 \text { in } \Omega\right\}
$$

and regard the following weak formulation under homogeneous Dirichlet boundary conditions with time-independent test functions: search for $\boldsymbol{u}(t) \in \mathcal{V}(\Omega)$ such that for all $\boldsymbol{v} \in \mathcal{V}(\Omega)$

$$
\frac{\mathrm{d}}{\mathrm{dt}}(\boldsymbol{u}(t), \boldsymbol{v})_{\Omega}+\mu(\nabla \boldsymbol{u}(t), \nabla \boldsymbol{v})_{\Omega}+((\boldsymbol{u}(t) \cdot \nabla) \boldsymbol{u}(t), \boldsymbol{v})_{\Omega}=\langle\boldsymbol{f}(t), \boldsymbol{v}\rangle_{\Omega}
$$

holds, in the sense of distributions in $\mathcal{D}^{\prime}(] 0, T[)$, with $\boldsymbol{u}(0)=\boldsymbol{u}_{0}$ in $\mathcal{V}(\Omega)^{\prime}$ fulfilled in the weak sense; see [9]. We remark that the weak formulation is pressure-free, avoiding issues with a possible low regularity of the pressure field in the transient nonlinear setting.

Let now $\boldsymbol{f}_{1}, \boldsymbol{f}_{2} \in H^{-1}(\Omega)^{d}$ be two forcings differing only by a gradient field, i.e., $\boldsymbol{f}_{1}-\boldsymbol{f}_{2}=\nabla \phi$ with $\phi \in L^{2}(\Omega)$. We interpret these forcings as functionals in $\mathcal{V}(\Omega)^{\prime}$ and compute for arbitrary $\boldsymbol{v} \in \mathcal{V}(\Omega)$

$$
\left\langle\boldsymbol{f}_{1}, \boldsymbol{v}\right\rangle_{\Omega}-\left\langle\boldsymbol{f}_{2}, \boldsymbol{v}\right\rangle_{\Omega}=\left\langle\boldsymbol{f}_{1}-\boldsymbol{f}_{2}, \boldsymbol{v}\right\rangle_{\Omega}=-(\phi, \nabla \cdot \boldsymbol{v})_{\Omega}=0 .
$$

Thus, $\boldsymbol{f}_{1}$ and $\boldsymbol{f}_{2}$ are identical if they are regarded as functionals in $\mathcal{V}(\Omega)^{\prime}$. This leads to the fundamental observation that $\boldsymbol{f}_{1}$ and $\boldsymbol{f}_{2}$ are velocity-equivalent in the sense that they induce the very same velocity solution in (1.4). A difference between $\boldsymbol{f}_{1}$ and $\boldsymbol{f}_{2}$ can only be recognized in the original equations (1.1), where the different forcings would lead to pressure gradients differing exactly by $\nabla \phi$. Thus, the notion of velocity equivalence of two functionals in $H^{-1}(\Omega)^{d}$ can be formally defined by

$$
\boldsymbol{f}_{1} \simeq \boldsymbol{f}_{2} \quad: \Leftrightarrow \quad \exists q \in L_{0}^{2}(\Omega): \forall \boldsymbol{w} \in H_{0}^{1}(\Omega)^{d}\left\langle\boldsymbol{f}_{1}-\boldsymbol{f}_{2}, \boldsymbol{w}\right\rangle_{\Omega}=-(q, \nabla \cdot \boldsymbol{w})_{\Omega} .
$$

The corresponding seminorm, which induces these equivalence classes of functionals, is naturally given for $f \in H^{-1}(\Omega)^{d}$ by

$$
\|\boldsymbol{f}\|_{\mathcal{v}(\Omega)^{\prime}}:=\sup _{\mathbf{0} \neq \boldsymbol{v} \in \mathcal{V}(\Omega)} \frac{\left|\langle\boldsymbol{f}, \boldsymbol{v}\rangle_{\Omega}\right|}{\|\nabla \boldsymbol{v}\|_{0, \Omega}} .
$$

Clearly, the above supremum is a seminorm since $\|\nabla \phi\|_{\mathcal{v}(\Omega)^{\prime}}=0$ for all $\phi \in L^{2}(\Omega)$.

Turning back to the issue of constructing discrete stabilization operators for dominant advection in Navier-Stokes flows, we remark that also the strength of the advection term has to be measured in the seminorm (1.6), and not in the standard $H^{-1}(\Omega)^{d}$-norm. Seeing things from this angle, we see that a nonzero convective term lies in between the following two extreme cases:

(1) a gradient field, leading to no dominant advection in the sense above due to $\|(\boldsymbol{u} \cdot \nabla) \boldsymbol{u}\|_{\mathcal{v}(\Omega)^{\prime}}=0$

(2) a divergence-free field, leading to dominant advection.

In the first extreme case (i.e., (1) above), where $\|(\boldsymbol{u} \cdot \nabla) \boldsymbol{u}\|_{-1, \Omega}$ is large, although it holds that $\|(\boldsymbol{u} \cdot \nabla) \boldsymbol{u}\|_{\mathcal{v}(\Omega)^{\prime}}=0$, pressure-robust mixed methods have been shown recently to outperform classical mixed methods that are only LBB-stable [33, 28]. 
They are designed in such a way that any gradient forcing in $\left(\boldsymbol{u}_{h} \cdot \nabla\right) \boldsymbol{u}_{h}$ does not change the discrete velocity solution $\boldsymbol{u}_{h}$, respecting on the discrete level the equivalence classes that are induced by the seminorm (1.6). From a more applied point of view, pressure-robust methods have been shown to be important for vortex-dominated flows $[33,58]$, where the following relation between the convective term and the pressure gradient holds

$$
(\boldsymbol{u} \cdot \nabla) \boldsymbol{u}+\nabla p \approx \mathbf{0}
$$

this means that the centrifugal force within the vortex structure is balanced by the pressure gradient. Such flows are known as generalized Beltrami flows and are intensively studied in topological fluid dynamics (cf. [5]), and they are popular benchmark problems. For this type of flow, due to (1.7) the quadratic velocity-dependent convection term balances the linear pressure gradient, and then the pressure field is usually more complicated than the velocity field. As a consequence, it has been demonstrated numerically for this class of time-dependent high Reynolds number flows that pressure-robust DG methods of order $k$ delivered on coarse grids similarly accurate results as DG methods of order $2 k$ that are only LBB-stable [33].

With respect to (2) above, in order to derive an appropriate convection stabilization for the divergence-free part of $(\boldsymbol{u} \cdot \nabla) \boldsymbol{u}$, actually measured by the seminorm $\|(\boldsymbol{u} \cdot \nabla) \boldsymbol{u}\|_{\mathcal{V}(\Omega)^{\prime}}$, we try to obtain a better intuition for the meaning of the weak formulation (1.4). Exploiting that every divergence-free function $\boldsymbol{v} \in \mathcal{V}(\Omega)$ has a vector potential $\boldsymbol{v}=\operatorname{curl} \boldsymbol{\chi}$ [34], we can formally derive for smooth enough functionals $\boldsymbol{f}$

$$
(\boldsymbol{f}, \boldsymbol{v})_{\Omega}=(\boldsymbol{f}, \operatorname{curl} \boldsymbol{\chi})_{\Omega}=(\operatorname{curl} \boldsymbol{f}, \boldsymbol{\chi})_{\Omega} .
$$

When applied to the term $\boldsymbol{u}_{t}$, a similar integration by parts with the curl operator and introducing the vorticity $\boldsymbol{\omega}:=\operatorname{curl} \boldsymbol{u}$ will yield

$$
\frac{\mathrm{d}}{\mathrm{dt}}(\boldsymbol{u}(t), \boldsymbol{v})_{\Omega}=\frac{d}{\mathrm{dt}}(\boldsymbol{u}(t), \operatorname{curl} \boldsymbol{\chi})_{\Omega}=\frac{d}{\mathrm{dt}}(\boldsymbol{\omega}(t), \boldsymbol{\chi})_{\Omega},
$$

and applying the same idea to the remaining terms in (1.4) reveals that the weak formulation (1.4) can be understood as a mathematically precise formulation of the vorticity equation

$$
\boldsymbol{\omega}_{t}-\mu \Delta \boldsymbol{\omega}+(\boldsymbol{u} \cdot \nabla) \boldsymbol{\omega}-(\boldsymbol{\omega} \cdot \nabla) \boldsymbol{u}=\operatorname{curl} \boldsymbol{f} ;
$$

cf. $[42,19]$. In this last equation, the gradient of the pressure has completely disappeared. So, starting from this remark in this work we propose a residual-based stabilization of the vorticity equation, which we call least squares vorticity stabilization (LSVS). This stabilization strategy includes a higher order stabilization term on the vorticity equation in the bulk and a penalty on the jump of the tangential component of the convective derivative over element faces (see section 2.2 for details). A similar starting point was used in the meteorology community [52], where a residual SUPG-like method built from (1.8) for the two-dimensional case (although different from the one proposed in this work, and no analysis was presented). The same principle has also been applied in recent work on pressure-robust residual-based a posteriori error control [46, 43].

To keep the technical details down we restrict the analysis to a linearized problem, namely the following Oseen's problem on a bounded, connected, contractible, polyhedral Lipschitz domain $\Omega$ : 


$$
\begin{aligned}
& \mathcal{L} \boldsymbol{u}+\nabla p=\boldsymbol{f} \quad \text { in } \Omega, \\
& \operatorname{div} \boldsymbol{u}=0 \quad \text { in } \Omega, \\
& \boldsymbol{u}=\mathbf{0} \quad \text { on } \partial \Omega,
\end{aligned}
$$

where

$$
\mathcal{L} \boldsymbol{u}:=\sigma \boldsymbol{u}+(\boldsymbol{\beta} \cdot \nabla) \boldsymbol{u}-\mu \Delta \boldsymbol{u} .
$$

Here, $\mu>0$ denotes the diffusion coefficient, $\sigma>0$, and the convective term $\boldsymbol{\beta}$ is assumed to belong to $W^{1, \infty}(\Omega)^{d}$ and to satisfy $\operatorname{div} \boldsymbol{\beta}=0$. This is an elliptic system that is well posed in $H_{0}^{1}(\Omega)^{d} \cap \mathcal{V}(\Omega) \times L_{0}^{2}(\Omega)$ by the Lax-Milgram lemma and Brezzi's theorem for all $\mu>0$. A weak formulation of Oseen's problem, which is in the spirit of the weak formulation (1.4) for the time-dependent incompressible Navier-Stokes equations, is given by, Find $\boldsymbol{u} \in \mathcal{V}(\Omega)$ such that for all $\boldsymbol{v} \in \mathcal{V}(\Omega)$ the following holds:

$$
\mu(\nabla \boldsymbol{u}, \nabla \boldsymbol{v})_{\Omega}+((\boldsymbol{\beta} \cdot \nabla) \boldsymbol{u}, \boldsymbol{v})_{\Omega}+\sigma(\boldsymbol{u}, \boldsymbol{v})_{\Omega}=\langle\boldsymbol{f}, \boldsymbol{v}\rangle_{\Omega} .
$$

In the following, we will refer to this weak formulation as Oseen's problem. We will nevertheless always keep in mind that, given the unique solution of (1.11), there exists a unique pressure $p \in L_{0}^{2}(\Omega)$ such that $(\boldsymbol{u}, p)$ satisfies the mixed weak formulation of (1.9).

1.2. Preliminary results. In this work we follow the convention given in, e.g., [34, p. 31], where for $d=2$ and a scalar function $z$, its vector-valued curl is given by

$$
\operatorname{curl} z=\left(\partial_{y} z,-\partial_{x} z\right) \text {. }
$$

As already mentioned, for every divergence-free function in $\Omega$ we can associate a potential (scalar-valued for $d=2$ and vector-valued for $d=3$ ). So, natural spaces to consider that can capture the kernel of the divergence operator are given by

$$
Z:= \begin{cases}\left\{z \in H^{1}(\Omega)^{3}: \operatorname{curl} z \in H_{0}^{1}(\Omega)^{3}\right\} & \text { if } d=3, \\ \left\{z \in H^{1}(\Omega): \operatorname{curl} z \in H_{0}^{1}(\Omega)^{2}\right\} & \text { if } d=2 .\end{cases}
$$

We stress the fact that for $d=2, z$ is a scalar function, while for $d=3, z$ is a vector-valued function. To simplify the presentation from now on we will just use the boldface notation for both cases, and the definition will depend on the context.

Using the generalized Bogovskii operator since $\Omega$ is contractible and Lipschitz there exists $\boldsymbol{z}$ with components in $H_{0}^{2}(\Omega)[24]$ such that

$$
\operatorname{curl} \boldsymbol{z}=\boldsymbol{u} \quad \text { in } \Omega \text {. }
$$

It is important to notice here that $\boldsymbol{z}$ and its first derivative vanish on $\partial \Omega$. If we assume more regularity of $\boldsymbol{u}$, then we can find a smoother $\boldsymbol{z}$ satisfying (1.13); however, it may not satisfy boundary conditions. More precisely, the following result is a rewriting of $[24$, Theorem 4.9(b)], where we have used that, since the domain $\Omega$ is supposed to be contractible, then the cohomology space is zero.

Proposition 1. Let $\Omega \subset \mathbb{R}^{d}$ be a contractible, Lipschitz polygonal/polyhedral domain. Let $\boldsymbol{u} \in H^{r}(\Omega)^{d}$ with $r \geq 1$ such that $\operatorname{div} \boldsymbol{u}=0$. Then, there exists $\boldsymbol{z}$ with components in $H^{r+1}(\Omega)$ satisfying (1.13) and the following stability estimate:

$$
\|\boldsymbol{z}\|_{r+1, \Omega} \leq C\|\boldsymbol{u}\|_{r, \Omega},
$$

where the constant $C>0$ is independent of $\boldsymbol{u}$.

Copyright $@$ by SIAM. Unauthorized reproduction of this article is prohibited. 
Note that the boundary conditions $\boldsymbol{z}=0$ on $\partial \Omega$ might not hold even if $\boldsymbol{u}$ vanishes on $\partial \Omega$ if we would like $r \geq 2$. However, in two dimensions we can guarantee that boundary conditions are satisfied.

Corollary 2. Under the hypotheses of Proposition 1, if $d=2$ we can choose $\boldsymbol{z}$ satisfying (1.13) and (1.14), so that $\boldsymbol{z}=0$ on $\partial \Omega$.

Proof. Let us assume that $d=2$. By Proposition 1 there exists $z \in H^{r+1}(\Omega)$ so that (1.13) and (1.14) hold. Since curl $\boldsymbol{z}=\boldsymbol{u}$ we have that curl $\boldsymbol{z}=\mathbf{0}$ on $\partial \Omega$. Denoting by $\boldsymbol{t}$ the unit tangent vector to $\partial \Omega$, this implies that $\nabla \boldsymbol{z} \cdot \boldsymbol{t}=0$ on $\partial \Omega$ and then $\boldsymbol{z}$ is constant on $\partial \Omega$. Let us denote that constant $c \in \mathbb{R}$. Then, the function $\tilde{\boldsymbol{z}}=\boldsymbol{z}-c$ satisfies all the requirements of the result, including estimate (1.14).

\section{The stabilized finite element method.}

2.1. Finite element spaces. Let $\left\{\mathcal{T}_{h}\right\}_{h>0}$ be a family of shape-regular simplicial triangulations of $\Omega$. The elements of $\mathcal{T}_{h}$ will be denoted by $K$ with diameter $h_{K}:=\operatorname{diam}(K)$ and maximal mesh width $h=\max \left\{h_{K}: K \in \mathcal{T}_{h}\right\}$. For an element $K \in \mathcal{T}_{h}$, we define the set $\mathcal{F}_{K}$ of its facets. The set of all facets of the triangulation $\mathcal{T}_{h}$ is denoted by $\mathcal{F}$ and $\mathcal{F}^{i}$ denotes the interior facets. For $F \in \mathcal{F}$ we will denote $h_{F}=\operatorname{diam}(F)$, and $|F|$ the $(d-1)$-dimensional measure of $F$ (area for $d=3$ and length for $d=2$ ). The $L^{2}(F)$-inner product is denoted by $\langle\cdot, \cdot\rangle_{F}$. For a vector-valued function $\boldsymbol{v}$ we define the tangential jumps across $F=K_{1} \cap K_{2}$ with $K_{1}, K_{2} \in \mathcal{T}_{h}$ as

$$
\llbracket \boldsymbol{v} \times\left.\boldsymbol{n} \rrbracket\right|_{F}:=\boldsymbol{v}_{1} \times \boldsymbol{n}_{1}+\boldsymbol{v}_{2} \times \boldsymbol{n}_{2},
$$

where $\boldsymbol{v}_{i}=\left.\boldsymbol{v}\right|_{K_{i}}$ and $\boldsymbol{n}_{i}$ is the unit normal pointing out of $K_{i}$. If $F$ is a boundary face, then we define

$$
\llbracket \boldsymbol{v} \times\left.\boldsymbol{n} \rrbracket\right|_{F}:=\boldsymbol{v} \times \boldsymbol{n} .
$$

In addition, we introduce the following broken inner products (assuming the functions involved are regular enough so every quantity is finite):

$$
(v, w)_{h}:=\sum_{K \in \mathcal{T}_{h}}(v, w)_{K}, \quad\langle v, w\rangle_{\mathcal{F}^{i}}:=\sum_{F \in \mathcal{F}^{i}}\langle v, w\rangle_{F} \quad \text { and } \quad\langle v, w\rangle_{\mathcal{F}}:=\sum_{F \in \mathcal{F}}\langle v, w\rangle_{F}
$$

with associated norms $\|\cdot\|_{h},\|\cdot\|_{h, \mathcal{F}},\|\cdot\|_{h, \mathcal{F}^{i}}$, respectively.

For $s \geq 1$ we define the standard piecewise polynomial Lagrange space by

$$
\boldsymbol{W}_{h}^{s}:=\left\{\boldsymbol{w} \in H_{0}^{1}(\Omega)^{d}:\left.\boldsymbol{w}\right|_{K} \in \mathbb{P}_{s}(K)^{d} \quad \forall K \in \mathcal{T}_{h}\right\} .
$$

Over $\mathcal{T}_{h}$, and for $k \geq 1$, we assume we have finite element spaces $\boldsymbol{V}_{h} \subset H_{0}^{1}(\Omega)^{d}$, $Q_{h} \subset L_{0}^{2}(\Omega)$ and the associated subspace of (exactly) divergence-free functions

$$
\mathcal{V}_{h}:=\left\{\boldsymbol{v}_{h} \in \boldsymbol{V}_{h}: \text { such that } \operatorname{div} \boldsymbol{v}_{h}=0 \text { in } \Omega\right\},
$$

satisfying the following assumptions:

(A1) $\operatorname{div} \boldsymbol{V}_{h} \subset Q_{h}$;

(A2) the pair $\left(\boldsymbol{V}_{h}, Q_{h}\right)$ is inf-sup stable;

(A3) $\boldsymbol{W}_{h}^{k} \subset \boldsymbol{V}_{h} \subset \boldsymbol{W}_{h}^{r}$ for some $r, k \geq 1$;

(A4) there exists a finite element space $\boldsymbol{Z}_{h} \subset \boldsymbol{Z}$ such that $\operatorname{curl} \boldsymbol{Z}_{h}=\mathcal{V}_{h}$; 
(A5) any $\boldsymbol{z} \in \boldsymbol{Z}$ with components in $H^{k+2}(\Omega)$ satisfies the following estimate: for every multi-index $\boldsymbol{\alpha}=\left(\alpha_{1}, \ldots, \alpha_{d}\right) \in \mathbb{R}^{d}$, where $|\boldsymbol{\alpha}|:=\alpha_{1}+\cdots+\alpha_{d}$, the following approximation holds:

$$
\inf _{\boldsymbol{\psi}_{h} \in \boldsymbol{Z}_{h}}\left\|h^{|\boldsymbol{\alpha}|} \partial^{\boldsymbol{\alpha}}\left(\boldsymbol{z}-\boldsymbol{\psi}_{h}\right)\right\|_{h} \leq C h^{k+2}\|\boldsymbol{z}\|_{k+2, \Omega} \quad \text { for }|\boldsymbol{\alpha}| \leq k+1 ;
$$

(A6) if $d=2$ we can choose $\boldsymbol{Z}_{h}$ so that $\boldsymbol{Z}_{h} \subset H_{0}^{1}(\Omega)$.

Remark 3. We finish this section by giving an alternative interpretation of (A4)(A5). In fact, (A4)-(A5) imply that the space $\mathcal{V}(\Omega)$ can be approximated by functions in $\mathcal{V}_{h}$ in the sense that for all $\boldsymbol{v} \in \mathcal{V}(\Omega)$, the following approximation result holds:

$$
\inf _{\boldsymbol{w}_{h} \in \mathcal{V}_{h}}\left\|\nabla \boldsymbol{v}-\nabla \boldsymbol{w}_{h}\right\|_{0, \Omega} \leq\left(1+C_{F}\right) \inf _{\boldsymbol{w}_{h} \in \boldsymbol{V}_{h}}\left\|\nabla \boldsymbol{v}-\nabla \boldsymbol{w}_{h}\right\|_{0, \Omega}
$$

Here, $C_{F}$ denotes the stability constant of a Fortin operator, whose existence is ensured by LBB-stability; see [42, 8,34$]$.

2.1.1. Examples of finite element methods satisfying (A1)-(A6). Assumptions (A1)-(A6) essentially state that the finite element spaces are piecewise polynomials (so inverse inequalities are valid) and that the space $\mathcal{V}(\Omega)$ can be approximated, with optimal order, by the space $\mathcal{V}_{h}$. In addition, they state that the space of vector potentials associated to the space $\mathcal{V}(\Omega)$ can also be approximated, with optimal order, by the space $\boldsymbol{Z}_{h}$ containing the discrete vector potentials. This last hypothesis will be vital in the error analysis. We now present a few examples of finite element spaces that satisfy assumptions (A1)-(A6). The most classical example (and the one we use in our numerical experiments) is the Scott-Vogelius element [59], where

$$
\boldsymbol{V}_{h}=\boldsymbol{W}_{h}^{k} \quad \text { and } \quad Q_{h}=\left\{q_{h} \in L_{0}^{2}(\Omega):\left.q_{h}\right|_{K} \in \mathbb{P}_{k-1}(K) \forall K \in \mathcal{T}_{h}\right\} .
$$

The Scott-Vogelius element is LBB-stable on different kinds of shape-regular triangulations for different kinds of polynomial orders. For example, on shape-regular, barycentrically refined meshes, the condition $k \geq d$ suffices [54, 63, 39]. For $d=2$, the condition $k \geq 4$ allows us to derive LBB-stability on rather general, shape-regular meshes $[40,59]$, potentially modifying the pressure space to allow singular vertices. Characterizing the discrete potential space $\boldsymbol{Z}_{h}$ for the above examples has been addressed in several papers $[27,32]$ and they usually form an exact sequence. In particular, the space $\boldsymbol{Z}_{h}$ in the case $d=2$ on barycentrically refined meshes is the CloughTocher $C^{1}$ space [22]. Additional exact sequences, possibly using even smoother spaces, that lead to spaces satisfying our assumptions can be found in [36, 21, 27, 53].

In addition, it is worth mentioning that the requirement (A3), stating that the functions used to approximate the velocity are piecewise polynomials, prevents us from using spaces with rational functions, such as the ones proposed in [38, 37]. Nevertheless, the same analysis carried out below can be applied, with minor modifications, to that case as well. The same observation can be made about methods that belong to the IGA family proposed in, e.g., [13, 26, 25], since they are built using smooth rational functions, rather than polynomials.

2.2. The method. The idea is to remove all the gradient fields from the momentum equations in the stabilization, including the pressure gradient, by adding stabilization only on the vorticity equation instead of the velocity-pressure one, since any gradient is in the kernel of the curl operator. This amounts to adding a least 
squares term of the vorticity equation $\operatorname{curl} \mathcal{L} \boldsymbol{u}=\operatorname{curl} \boldsymbol{f}$. We multiply this equation by $\tau \operatorname{curl} \mathcal{L} \boldsymbol{v}$, where $\tau$ is a stabilization parameter chosen so that the stabilizing term scales in the same way as the equation (see (2.10) below). This leads to the term

$$
(\tau \operatorname{curl} \mathcal{L} \boldsymbol{u}, \operatorname{curl} \mathcal{L} \boldsymbol{v})_{h}=(\tau \operatorname{curl} \boldsymbol{f}, \operatorname{curl} \mathcal{L} \boldsymbol{v})_{h},
$$

or

$$
(\tau \operatorname{curl} \mathcal{L} \boldsymbol{u}, \operatorname{curl}(\boldsymbol{\beta} \cdot \nabla) \boldsymbol{v})_{h}=(\tau \operatorname{curl} \boldsymbol{f}, \operatorname{curl}(\boldsymbol{\beta} \cdot \nabla) \boldsymbol{v})_{h} .
$$

For simplicity we only consider the former form for the analysis below. Observe that this is a high order term, which for smooth flows can be assumed to be of a smaller magnitude than the boundary penalty term introduced next. In fact, if no further assumptions are made on the velocity space it is not sufficient to guarantee optimal bounds. Thus, a further control on the jumps of the convective gradients over the facets, similar to that proposed in [16], needs to be added to the formulation. So, on each internal facet $F$ we add the term

$$
\left\langle h^{2} \llbracket(\boldsymbol{\beta} \cdot \nabla) \boldsymbol{u}_{h} \times \boldsymbol{n} \rrbracket, \llbracket(\boldsymbol{\beta} \cdot \nabla) \boldsymbol{v}_{h} \times \boldsymbol{n} \rrbracket\right\rangle_{F} .
$$

Gathering the terms introduced above, the stabilized finite element method analyzed in this work reads, Find $\left(\boldsymbol{u}_{h}, p_{h}\right) \in \boldsymbol{V}_{h} \times Q_{h}$ such that

$$
\left\{\begin{aligned}
a\left(\boldsymbol{u}_{h}, \boldsymbol{v}_{h}\right)+S\left(\boldsymbol{u}_{h}, \boldsymbol{v}_{h}\right)-b\left(p_{h}, \boldsymbol{v}_{h}\right)= & L\left(\boldsymbol{v}_{h}\right) & & \forall \boldsymbol{v}_{h} \in \boldsymbol{V}_{h}, \\
b\left(q_{h}, \boldsymbol{u}_{h}\right)= & 0 & & \forall q_{h} \in Q_{h},
\end{aligned}\right.
$$

where the bilinear forms are defined by

$$
\begin{aligned}
a\left(\boldsymbol{u}_{h}, \boldsymbol{v}_{h}\right) & :=\left(\sigma \boldsymbol{u}_{h}+(\boldsymbol{\beta} \cdot \nabla) \boldsymbol{u}_{h}, \boldsymbol{v}_{h}\right)_{\Omega}+\mu\left(\nabla \boldsymbol{u}_{h}, \nabla \boldsymbol{v}_{h}\right)_{\Omega}, \\
b\left(p_{h}, \boldsymbol{v}_{h}\right) & :=\left(p_{h}, \nabla \cdot \boldsymbol{v}_{h}\right)_{\Omega},
\end{aligned}
$$

and the stabilizing bilinear form is given by

$$
S\left(\boldsymbol{u}_{h}, \boldsymbol{v}_{h}\right):=\delta_{0}\left\{\left(\tau \operatorname{curl} \mathcal{L} \boldsymbol{u}_{h}, \operatorname{curl} \mathcal{L} \boldsymbol{v}_{h}\right)_{h}+\left\langle h^{2} \llbracket(\boldsymbol{\beta} \cdot \nabla) \boldsymbol{u}_{h} \times \boldsymbol{n} \rrbracket, \llbracket(\boldsymbol{\beta} \cdot \nabla) \boldsymbol{v}_{h} \times \boldsymbol{n} \rrbracket\right\rangle_{\mathcal{F}^{i}}\right\} .
$$

Here the broken scalar products are defined in (2.1), and the stabilization parameter $\left.\tau\right|_{K}=\tau_{K}$ is given by

$$
\tau_{K}:=\min \left\{1, \frac{\|\boldsymbol{\beta}\|_{0, \infty, \Omega} h_{K}}{\mu}\right\} \frac{h_{K}^{3}}{\|\boldsymbol{\beta}\|_{0, \infty, \Omega}} .
$$

Finally, the right-hand-side $L$ is given by

$$
L\left(\boldsymbol{v}_{h}\right):=\left(\boldsymbol{f}, \boldsymbol{v}_{h}\right)_{\Omega}+\delta_{0}\left(\tau \operatorname{curl} \boldsymbol{f}, \operatorname{curl} \mathcal{L} \boldsymbol{v}_{h}\right)_{h} .
$$

In the stabilizing terms, $\delta_{0}>0$ is a nondimensional parameter. The value of $\delta_{0}$ does not affect the qualitative behavior of the error estimates, so we will not track this constant in our error estimates below. Nevertheless, in section 4 we will carry out a study of its optimal value.

For the analysis we introduce the following mesh-dependent norm:

$$
\|\boldsymbol{v}\|^{2}:=\left\|\sigma^{\frac{1}{2}} \boldsymbol{v}\right\|_{0, \Omega}^{2}+\left\|\mu^{\frac{1}{2}} \nabla \boldsymbol{v}\right\|_{0, \Omega}^{2}+|\boldsymbol{v}|_{S}^{2},
$$

Copyright (c) by SIAM. Unauthorized reproduction of this article is prohibited. 
where $|\boldsymbol{v}|_{S}^{2}:=S(\boldsymbol{v}, \boldsymbol{v})$. We see that

$$
\left\|\boldsymbol{v}_{h}\right\|^{2}=(a+S)\left(\boldsymbol{v}_{h}, \boldsymbol{v}_{h}\right) \quad \forall \boldsymbol{v}_{h} \in \boldsymbol{V}_{h} .
$$

In addition, the pair $\boldsymbol{V}_{h} \times Q_{h}$ satisfies the inf-sup condition, by assumption (A2), which ensures the well-posedness of problem (2.6). Moreover, method (2.6) is strongly consistent for smooth enough $(\boldsymbol{u}, p)$, that is,

$$
\left\{\begin{aligned}
a\left(\boldsymbol{u}-\boldsymbol{u}_{h}, \boldsymbol{v}_{h}\right)+S\left(\boldsymbol{u}-\boldsymbol{u}_{h}, \boldsymbol{v}_{h}\right)-b\left(p-p_{h}, \boldsymbol{v}_{h}\right)= & 0 \quad \forall \boldsymbol{v}_{h} \in \boldsymbol{V}_{h}, \\
b\left(q_{h}, \boldsymbol{u}-\boldsymbol{u}_{h}\right)= & 0 \quad \forall q_{h} \in Q_{h} .
\end{aligned}\right.
$$

Remark 4. We remark that method (2.6) can be also written, equivalently, in the following compact form: Find $\boldsymbol{u}_{h} \in \mathcal{V}_{h}$ such that

$$
a\left(\boldsymbol{u}_{h}, \boldsymbol{v}_{h}\right)+S\left(\boldsymbol{u}_{h}, \boldsymbol{v}_{h}\right)=L\left(\boldsymbol{v}_{h}\right) \quad \forall \boldsymbol{v}_{h} \in \mathcal{V}_{h} .
$$

This simplified form may be chosen for the analysis, as it does not involve the discrete pressure. However, we prefer to write (2.6) involving both pressure and velocity, as (2.15) cannot be implemented in an easy way, due to the necessity to identify the exactly divergence-free space $\mathcal{V}_{h}$ and its basis functions. This task is, in general, not straightforward.

Remark 5. In case of classical LBB-stable methods like Taylor-Hood, BernardiRaugel, or the mini elements, a similar approach employing the corresponding space of discretely divergence-free vector fields (still denoted by $\mathcal{V}_{h}$, but note that its elements are no longer exactly divergence-free) would lead to, For all $\boldsymbol{v}_{h} \in \mathcal{V}_{h}$ it holds that

$$
a\left(\boldsymbol{u}-\boldsymbol{u}_{h}, \boldsymbol{v}_{h}\right)+S\left(\boldsymbol{u}-\boldsymbol{u}_{h}, \boldsymbol{v}_{h}\right)=-\left(\nabla p, \boldsymbol{v}_{h}\right)_{\Omega},
$$

i.e., a consistency error of the form $-\left(\nabla p, \boldsymbol{v}_{h}\right)_{\Omega}$ appears. Introducing the notion of a discrete Helmholtz-Hodge projector $\mathbb{P}_{h}[49,48]$ as the $L^{2}(\Omega)$-projection onto the space of discretely divergence-free vector fields $\mathcal{V}_{h}$, one recognizes that this consistency error quantifies nothing other than the strength of this discrete Helmholtz-Hodge projector. Note that the continuous Helmholtz-Hodge projector of any gradient field $\nabla \phi \in L^{2}(\Omega)^{d}$ is zero, i.e., it has a very similar meaning as $\operatorname{curl} \nabla \phi=\mathbf{0}$; see [42]. For an LBB-stable method with a discrete pressure space with elementwise polynomials of order $k_{p}$, it is a classical result that the discrete Helmholtz-Hodge projector of any smooth gradient fields vanishes with order $k_{p}+1$ in the following discrete $\mathcal{V}_{h}^{\prime}$-norm (which can be interpreted as a $H^{-1}(\Omega)^{d}$ seminorm):

$$
\sup _{\mathbf{0} \neq \boldsymbol{v}_{h} \in \boldsymbol{V}_{h}} \frac{\left|\left(\nabla \phi, \boldsymbol{v}_{h}\right)_{\Omega}\right|}{\left\|\nabla \boldsymbol{v}_{h}\right\|_{0, \Omega}} \leq C h^{k_{p}+1}|\phi|_{k_{p}, \Omega} .
$$

But if one estimates the strength of the discrete Helmholtz-Hodge projector in a dual seminorm linked to $L^{2}(\Omega)$, one only obtains

$$
\sup _{\mathbf{0} \neq \boldsymbol{v}_{h} \in \boldsymbol{V}_{h}} \frac{\left|\left(\nabla \phi, \boldsymbol{v}_{h}\right)_{\Omega}\right|}{\left\|\boldsymbol{v}_{h}\right\|_{0, \Omega}} \leq C h^{k_{p}}|\phi|_{k_{p}+1, \Omega} ;
$$

see [49]. We conjecture that (2.17) essentially explains why it has not been possible so far to get an improved convergence order $h^{k+\frac{1}{2}}$ for advection stabilization of different order LBB-stable methods like the Taylor-Hood or Bernardi-Raugel elements. The gradient fields on the momentum balance are responsible for this behavior. Note that the discrete Helmholtz-Hodge projector of any pressure-robust method vanishes for arbitrary gradient fields [49, 48], and thanks to the link between the mini element and equal order $\mathbb{P}_{1} \times \mathbb{P}_{1}$ elements, an improved $O\left(h^{k+\frac{1}{2}}\right)$ order for the velocity can also be proven for the former under advection stabilization. 
3. Analysis of the approximation error. The two following results are classical and will be used in the proof of our error estimates. The first is the following local trace inequality: there exists $C>0$ such that for all $K \in \mathcal{T}_{h}, F \in \mathcal{F}_{K}$, and all $v \in H^{1}(K)$,

$$
\|v\|_{0, F} \leq C\left(h_{K}^{-\frac{1}{2}}\|v\|_{0, K}+h_{K}^{\frac{1}{2}}|v|_{1, K}\right) .
$$

We also recall the following inverse inequality: for all $\ell, s, m \in \mathbb{N}$ such that $0 \leq \ell \leq$ $s \leq m$ and all $q \in \mathbb{P}_{m}(K)$ there exists $C>0$ such that

$$
|q|_{s, K} \leq C h_{K}^{\ell-s}|q|_{\ell, K} .
$$

Finally, as our main interest is to track the dependency of the error estimates on the viscosity $\mu$, in order to avoid unnecessary technicalities, we will not track their dependency on $\boldsymbol{\beta}$, or $\sigma$.

3.1. An error estimate for the velocity. In order to state the error estimates we define the following norm, for functions that are regular enough:

$$
\|\boldsymbol{z}\|_{\star}^{2}:=\|\operatorname{curl} \boldsymbol{z}\|^{2}+(h+\mu) \sum_{s=0}^{4} h^{2 s-4}\left\|D^{s} \boldsymbol{z}\right\|_{h}^{2} .
$$

Here, by $D^{s} \boldsymbol{z}$ we mean the tensor $\left(\partial^{\boldsymbol{\alpha}} \boldsymbol{z}\right)_{|\boldsymbol{\alpha}|=s}$, that is, gradient for $s=1$, Hessian matrix for $s=2$, etc. We start by proving a quasi-best approximation result with respect to this norm.

TheOREM 6. Let $\boldsymbol{u} \in H_{0}^{1}(\Omega)^{d} \cap H^{3}(\Omega)^{d}$ be the solution to (1.9) and let $\boldsymbol{z}$ be its corresponding potential given by Corollary 2. Let $\left(\boldsymbol{u}_{h}, p_{h}\right)$ be the solution of (2.6). If $d=3$ we assume, in addition, that $\boldsymbol{\beta} \cdot \boldsymbol{n}=0$ on $\partial \Omega$. Then, the following error estimate holds:

$$
\left\|\boldsymbol{u}-\boldsymbol{u}_{h}\right\| \mid \leq C\left\|\boldsymbol{z}-\boldsymbol{\psi}_{h}\right\|_{\star} \quad \forall \boldsymbol{\psi}_{h} \in \boldsymbol{Z}_{h} .
$$

The constant $C>0$ is independent of $h$ and $\mu$.

Proof. Let $\boldsymbol{e}=\boldsymbol{u}-\boldsymbol{u}_{h}$. We let $\boldsymbol{\psi}_{h} \in \boldsymbol{Z}_{h}$ be arbitrary and set $\boldsymbol{w}_{h}:=\operatorname{curl} \boldsymbol{\psi}_{h}$. We note that $\boldsymbol{w}_{h} \in \mathcal{V}_{h}$ and then, using the Galerkin orthogonality (2.14), we have

$$
\|\boldsymbol{e}\|^{2}=a\left(\boldsymbol{e}, \boldsymbol{u}-\boldsymbol{w}_{h}\right)+S\left(\boldsymbol{e}, \boldsymbol{u}-\boldsymbol{w}_{h}\right) .
$$

We bound the right-hand side of (3.5) term by term. For the rest of the proof, $\epsilon>0$ is arbitrary but will be chosen sufficiently small later. Using the Cauchy-Schwarz and Young's inequalities we see that

$$
S\left(\boldsymbol{e}, \boldsymbol{u}-\boldsymbol{w}_{h}\right) \leq \epsilon\|\boldsymbol{e}\|^{2}+C\left\|\boldsymbol{u}-\boldsymbol{w}_{h}\right\|^{2} .
$$

We rewrite the first term in (3.5) by adding and subtracting (elementwise) $\mu \Delta \boldsymbol{e}$ to obtain

$$
a\left(\boldsymbol{e}, \boldsymbol{u}-\boldsymbol{w}_{h}\right)=\left(\mathcal{L} \boldsymbol{e}, \boldsymbol{u}-\boldsymbol{w}_{h}\right)_{h}+\left(\mu \Delta \boldsymbol{e}, \boldsymbol{u}-\boldsymbol{w}_{h}\right)_{h}+\left(\mu \nabla \boldsymbol{e}, \nabla\left(\boldsymbol{u}-\boldsymbol{w}_{h}\right)\right)_{\Omega} .
$$

To bound the third term on the right-hand side of (3.7), proceeding as in (3.6) gives

$$
\left(\mu \nabla \boldsymbol{e}, \nabla\left(\boldsymbol{u}-\boldsymbol{w}_{h}\right)\right)_{\Omega} \leq \epsilon\|\boldsymbol{e}\|^{2}+C\left\|\boldsymbol{u}-\boldsymbol{w}_{h}\right\|^{2} .
$$


For the second term in (3.7) we add and subtract $\boldsymbol{w}_{h}$, use an inverse inequality and Young's inequalities, and arrive at

$$
\begin{aligned}
& \left(\mu \Delta \boldsymbol{e}, \boldsymbol{u}-\boldsymbol{w}_{h}\right)_{h}=\left(\mu \Delta\left(\boldsymbol{u}-\boldsymbol{w}_{h}\right), \boldsymbol{u}-\boldsymbol{w}_{h}\right)_{h}+\left(\mu \Delta\left(\boldsymbol{w}_{h}-\boldsymbol{u}_{h}\right), \boldsymbol{u}-\boldsymbol{w}_{h}\right)_{h} \\
\leq & \frac{1}{2}\left\|h \sqrt{\mu} \Delta\left(\boldsymbol{u}-\boldsymbol{w}_{h}\right)\right\|_{h}^{2}+\frac{1}{2}\left(1+\frac{1}{\epsilon}\right)\left\|h^{-1} \sqrt{\mu}\left(\boldsymbol{u}-\boldsymbol{w}_{h}\right)\right\|_{h}^{2}+\frac{\epsilon}{2}\left\|h \sqrt{\mu} \Delta\left(\boldsymbol{w}_{h}-\boldsymbol{u}_{h}\right)\right\|_{h}^{2} \\
\leq & C \mu\left(\left\|h \Delta\left(\boldsymbol{u}-\boldsymbol{w}_{h}\right)\right\|_{h}^{2}+\left\|\nabla\left(\boldsymbol{u}-\boldsymbol{w}_{h}\right)\right\|_{0, \Omega}^{2}+\left\|h^{-1}\left(\boldsymbol{u}-\boldsymbol{w}_{h}\right)\right\|_{h}^{2}\right)+C \frac{\epsilon}{2}\left\|\sqrt{\mu} \nabla\left(\boldsymbol{u}-\boldsymbol{u}_{h}\right)\right\|_{h}^{2} \\
\leq & C \mu \sum_{s=1}^{3} h^{2 s-4}\left\|D^{s}\left(\boldsymbol{z}-\boldsymbol{\psi}_{h}\right)\right\|_{h}^{2}+C \epsilon\|\sqrt{\mu} \nabla \boldsymbol{e}\|_{h}^{2} \\
\leq & C\left\|\boldsymbol{z}-\boldsymbol{\psi}_{h}\right\|_{\star}^{2}+C \epsilon\|\boldsymbol{e}\|^{2} .
\end{aligned}
$$

We are only left with the bound for the first term on the right-hand side of (3.7). First, integrating by parts we rewrite it as follows:

$$
\left(\mathcal{L} \boldsymbol{e}, \boldsymbol{u}-\boldsymbol{w}_{h}\right)_{h}=\left(\mathcal{L} \boldsymbol{e}, \operatorname{curl}\left(\boldsymbol{z}-\boldsymbol{\psi}_{h}\right)\right)_{h}=\left(\operatorname{curl} \mathcal{L} \boldsymbol{e}, \boldsymbol{z}-\boldsymbol{\psi}_{h}\right)_{h}+\left\langle\llbracket \mathcal{L} \boldsymbol{e} \times \boldsymbol{n} \rrbracket, \boldsymbol{z}-\boldsymbol{\psi}_{h}\right\rangle_{\mathcal{F}} .
$$

Applying the Cauchy-Schwarz and Young's inequalities leads to the following bound for the first term in the right-hand side of (3.8):

$$
\begin{aligned}
\left(\operatorname{curl} \mathcal{L} \boldsymbol{e}, \boldsymbol{z}-\boldsymbol{\psi}_{h}\right)_{h} & \leq \epsilon\left\|\tau^{\frac{1}{2}} \operatorname{curl} \mathcal{L} \boldsymbol{e}\right\|_{h}^{2}+C\left\|\tau^{-\frac{1}{2}}\left(\boldsymbol{z}-\boldsymbol{\psi}_{h}\right)\right\|_{h}^{2} \\
& \leq \epsilon\|\boldsymbol{e}\|^{2}+C\left\|\boldsymbol{z}-\boldsymbol{\psi}_{h}\right\|_{\star}^{2},
\end{aligned}
$$

where in the last step we used that $\left\|\tau^{-\frac{1}{2}}\left(\boldsymbol{z}-\boldsymbol{\psi}_{h}\right)\right\|_{h}^{2} \leq C\left\|\boldsymbol{z}-\boldsymbol{\psi}_{h}\right\|_{\star}^{2}$, independently of the value of $\mu$. Next, for $d=2$ we use that $\boldsymbol{z}-\boldsymbol{\psi}_{h}=\mathbf{0}$ on $\partial \Omega$ (which follows from assumption (A6)). In the case $d=3$ we decompose $\boldsymbol{\beta}=\boldsymbol{\beta} \cdot \boldsymbol{n} \boldsymbol{n}+(\boldsymbol{\beta}-\boldsymbol{\beta} \cdot \boldsymbol{n} \boldsymbol{n})=: \boldsymbol{\beta}_{n}+\boldsymbol{\beta}_{t}$. Since $\boldsymbol{\beta}_{t}$ is parallel to the boundary $\partial \Omega$, we have that $\boldsymbol{\beta}_{t} \cdot \nabla \boldsymbol{e}=0$ (since $\boldsymbol{e}=\mathbf{0}$ on $\partial \Omega$ ). So, using $\boldsymbol{e}=\mathbf{0}$ and $\boldsymbol{\beta}_{n}=0$ (if $d=3$ ) on $\partial \Omega$ we see that the second term is equal to

$$
\begin{aligned}
\left\langle\llbracket \mathcal{L} \boldsymbol{e} \times \boldsymbol{n} \rrbracket, \boldsymbol{z}-\boldsymbol{\psi}_{h}\right\rangle_{\mathcal{F}}= & \left\langle\llbracket(\boldsymbol{\beta} \cdot \nabla) \boldsymbol{e} \times \boldsymbol{n} \rrbracket, \boldsymbol{z}-\boldsymbol{\psi}_{h}\right\rangle_{\mathcal{F}^{i}}+\underbrace{\left\langle(\boldsymbol{\beta} \cdot \nabla) \boldsymbol{e} \times \boldsymbol{n}, \boldsymbol{z}-\boldsymbol{\psi}_{h}\right\rangle_{\partial \Omega}}_{=0} \\
& +\left\langle\llbracket-\mu \Delta \boldsymbol{e} \times \boldsymbol{n} \rrbracket, \boldsymbol{z}-\boldsymbol{\psi}_{h}\right\rangle_{\mathcal{F}} \\
= & \left\langle\llbracket(\boldsymbol{\beta} \cdot \nabla) \boldsymbol{e} \times \boldsymbol{n} \rrbracket, \boldsymbol{z}-\boldsymbol{\psi}_{h}\right\rangle_{\mathcal{F}^{i}}+\left\langle\llbracket-\mu \Delta \boldsymbol{e} \times \boldsymbol{n} \rrbracket, \boldsymbol{z}-\boldsymbol{\psi}_{h}\right\rangle_{\mathcal{F}} .
\end{aligned}
$$

To bound the first term we use Young's inquality and the local trace theorem (3.1) to get to

$$
\begin{aligned}
\left\langle\llbracket \boldsymbol{\beta} \cdot \nabla \boldsymbol{e} \times \boldsymbol{n} \rrbracket, \boldsymbol{z}-\boldsymbol{\psi}_{h}\right\rangle_{\mathcal{F}^{i}} & \leq \epsilon\|h \llbracket(\boldsymbol{\beta} \cdot \nabla) \boldsymbol{e} \times \boldsymbol{n} \rrbracket\|_{h, \mathcal{F}^{i}}^{2}+C\left\|h^{-1}\left(\boldsymbol{z}-\boldsymbol{\psi}_{h}\right)\right\|_{h, \mathcal{F}^{i}}^{2} \\
& \leq C \epsilon\|\boldsymbol{e}\|^{2}+C \sum_{s=0}^{1} h^{2 s-3}\left\|D^{s}\left(\boldsymbol{z}-\boldsymbol{\psi}_{h}\right)\right\|_{h}^{2} \\
& \leq C \epsilon\|\boldsymbol{e}\|^{2}+C\left\|\boldsymbol{z}-\boldsymbol{\psi}_{h}\right\|_{\star}^{2} .
\end{aligned}
$$

For the remaining term in (3.10) we add and subtract $\boldsymbol{w}_{h}$ and get

$$
\begin{aligned}
\left\langle\llbracket-\mu \Delta \boldsymbol{e} \times \boldsymbol{n} \rrbracket, \boldsymbol{z}-\boldsymbol{\psi}_{h}\right\rangle_{\mathcal{F}}=\langle\llbracket & \left.-\mu \Delta\left(\boldsymbol{u}-\boldsymbol{w}_{h}\right) \times \boldsymbol{n} \rrbracket, \boldsymbol{z}-\boldsymbol{\psi}_{h}\right\rangle_{\mathcal{F}} \\
& +\left\langle\llbracket-\mu \Delta\left(\boldsymbol{w}_{h}-\boldsymbol{u}_{h}\right) \times \boldsymbol{n} \rrbracket, \boldsymbol{z}-\boldsymbol{\psi}_{h}\right\rangle_{\mathcal{F}} .
\end{aligned}
$$

Copyright $\odot$ by SIAM. Unauthorized reproduction of this article is prohibited. 
To bound the first term of (3.11) we apply the Cauchy-Schwarz and Young's inequalities and the local trace result (3.1) to arrive at

$$
\begin{aligned}
& \left\langle\llbracket-\mu \Delta\left(\boldsymbol{u}-\boldsymbol{w}_{h}\right) \times \boldsymbol{n} \rrbracket, \boldsymbol{z}-\boldsymbol{\psi}_{h}\right\rangle_{\mathcal{F}} \\
\leq & \frac{1}{2}\left\|h^{3 / 2} \sqrt{\mu} \llbracket \Delta\left(\boldsymbol{u}-\boldsymbol{w}_{h}\right) \times \boldsymbol{n} \rrbracket\right\|_{h, \mathcal{F}}^{2}+\frac{1}{2}\left\|h^{-3 / 2} \sqrt{\mu}\left(\boldsymbol{z}-\boldsymbol{\psi}_{h}\right)\right\|_{h, \mathcal{F}}^{2} \\
\leq & C \mu\left(h^{2}\left\|\Delta\left(\boldsymbol{u}-\boldsymbol{w}_{h}\right)\right\|_{h}^{2}+h^{4}\left\|\nabla \Delta\left(\boldsymbol{u}-\boldsymbol{w}_{h}\right)\right\|_{h}^{2}+h^{-4}\left\|\boldsymbol{z}-\boldsymbol{\psi}_{h}\right\|_{h}^{2}+h^{-2}\left\|\nabla\left(\boldsymbol{z}-\boldsymbol{\psi}_{h}\right)\right\|_{h}^{2}\right) \\
\leq & C \mu \sum_{s=0}^{4} h^{2 s-4}\left\|D^{s}\left(\boldsymbol{z}-\boldsymbol{\psi}_{h}\right)\right\|_{h}^{2} \leq C\left\|\boldsymbol{z}-\boldsymbol{\psi}_{h}\right\|_{\star}^{2} .
\end{aligned}
$$

For the second term on (3.11) we use the Cauchy-Schwarz inequality, the local trace result (3.1), the inverse estimate (3.2), and Young's inequality, leading to

$$
\begin{aligned}
\left\langle\llbracket-\mu \Delta\left(\boldsymbol{w}_{h}-\boldsymbol{u}_{h}\right) \times \boldsymbol{n} \rrbracket, \boldsymbol{z}-\boldsymbol{\psi}_{h}\right\rangle_{\mathcal{F}} & \leq \epsilon\left\|\boldsymbol{w}_{h}-\boldsymbol{u}_{h}\right\|^{2}+C\left\|h^{-3 / 2} \sqrt{\mu}\left(\boldsymbol{z}-\boldsymbol{\psi}_{h}\right)\right\|_{h, \mathcal{F}}^{2} \\
& \leq 2 \epsilon\|\boldsymbol{e}\|^{2}+C\left\|\boldsymbol{z}-\boldsymbol{\psi}_{h}\right\|_{\star}^{2} .
\end{aligned}
$$

Hence, combining the above results and inserting the bounds into (3.5) gives

$$
\|\boldsymbol{e}\|^{2} \leq C \epsilon\|\boldsymbol{e}\|^{2}+C\left\|\boldsymbol{z}-\boldsymbol{\psi}_{h}\right\|_{\star}^{2} .
$$

Taking $\epsilon$ sufficiently small and rearranging terms finishes the proof.

The last result stresses the fact that the approximation of the solution depends only on how well the space $\boldsymbol{Z}_{h}$ approximates the space $\boldsymbol{Z}$, or, in other words, on how well the potential $\boldsymbol{z}$ is approximated by $\boldsymbol{Z}_{h}$. To make this bound more precise, we use assumption (A5) and Corollary 2 to obtain the following result.

Corollary 7. Let us assume, in addition to the hypotheses of Theorem 6 , that $\boldsymbol{u} \in H_{0}^{1}(\Omega)^{d} \cap H^{k+1}(\Omega)^{d}$. Then, there exists a constant $C>0$, independent of $h$ and $\mu$, such that

$$
\left\|\boldsymbol{u}-\boldsymbol{u}_{h}\right\| \leq C h^{k}\left(h^{\frac{1}{2}}+\mu^{\frac{1}{2}}\right)\|\boldsymbol{u}\|_{k+1, \Omega} .
$$

Two conclusions can be drawn from this last result. First, that method (2.6) has optimal, pressure-robust convergence rates. In addition, if the extra hypothesis $\mu \leq C h$ is imposed, then (3.12) leads to an $O\left(h^{k+\frac{1}{2}}\right)$ error estimate. This sort of estimate has only been obtained very recently for an incompressible problem using RT and BDM spaces in [6], and, to our best knowledge, the present result constitutes the first time such an estimate is obtained for stabilized methods for the Oseen equation using inf-sup stable elements with different approximation properties for the pressure and velocity spaces. We stress that the shape of the stabilization used is essential to obtain these results.

3.2. An error estimate for the pressure. For regular enough solutions (at least $H^{3}(\Omega)^{d}$ for the velocity), we will now show a supercloseness result for the discrete pressure that depends on the velocity error estimate only, which makes it pressurerobust. We denote by $\pi_{h}: L^{2}(\Omega) \rightarrow Q_{h}$ the $L^{2}(\Omega)$ orthogonal projection onto $Q_{h}$.

Thanks to the Galerkin orthogonality (2.14) and the fact that $\operatorname{div} \boldsymbol{V}_{h} \subseteq Q_{h}$ (see (A1)) we get, for an arbitrary $\boldsymbol{v}_{h} \in \boldsymbol{V}_{h}$,

$$
\begin{aligned}
a\left(\boldsymbol{u}-\boldsymbol{u}_{h}, \boldsymbol{v}_{h}\right)+S\left(\boldsymbol{u}-\boldsymbol{u}_{h}, \boldsymbol{v}_{h}\right) & =\left(p-p_{h}, \nabla \cdot \boldsymbol{v}_{h}\right)_{\Omega} \\
& =\left(\pi_{h} p-p_{h}, \nabla \cdot \boldsymbol{v}_{h}\right)_{\Omega} .
\end{aligned}
$$

Copyright (c) by SIAM. Unauthorized reproduction of this article is prohibited. 
The pair $\boldsymbol{V}_{h} \times Q_{h}$ is inf-sup stable (see (A2)), which guarantees the existence of a Fortin operator onto $Q_{h}$ that commutes with the divergence. Since in addition $\operatorname{div} \boldsymbol{V}_{h} \subseteq Q_{h}$ (see (A1)), then this operator is surjective. So, there exists an $\boldsymbol{x}_{h} \in \boldsymbol{V}_{h}$ such that

$$
\operatorname{div} \boldsymbol{x}_{h}=\pi_{h} p-p_{h} \quad \text { in } \Omega \quad \text { and } \quad\left\|\nabla \boldsymbol{x}_{h}\right\|_{0, \Omega} \leq C\left\|\pi_{h} p-p_{h}\right\|_{0, \Omega},
$$

where $C>0$ only depends on $\Omega$. Thus, integrating by parts, using that $\operatorname{div} \boldsymbol{\beta}=0$, and the Cauchy-Schwarz inequality, we arrive at

$$
\begin{aligned}
\left\|\pi_{h} p-p_{h}\right\|_{0, \Omega}^{2} & =a\left(\boldsymbol{u}-\boldsymbol{u}_{h}, \boldsymbol{x}_{h}\right)+S\left(\boldsymbol{u}-\boldsymbol{u}_{h}, \boldsymbol{x}_{h}\right) \\
& \leq\left\|\boldsymbol{u}-\boldsymbol{u}_{h}\right\| \cdot\left\|\boldsymbol{x}_{h}\right\|-\left((\boldsymbol{\beta} \cdot \nabla) \boldsymbol{x}_{h}, \boldsymbol{u}-\boldsymbol{u}_{h}\right)_{\Omega} \\
& \leq\left\|\boldsymbol{u}-\boldsymbol{u}_{h}\right\|\|\cdot\| \boldsymbol{x}_{h}\|+\| \boldsymbol{\beta}\left\|_{0, \infty, \Omega}\right\| \boldsymbol{u}-\boldsymbol{u}_{h}\left\|_{0, \Omega}\right\| \nabla \boldsymbol{x}_{h} \|_{0, \Omega} .
\end{aligned}
$$

Thanks to the stability result in (3.13), once the bound $\left\|\boldsymbol{x}_{h}\right\| \leq C\left\|\pi_{h} p-p_{h}\right\|_{0, \Omega}$ is established, then (3.14) provides an error estimate for $\pi_{h} p-p_{h}$ in terms of the velocity error estimate only. So, it only remains to bound the triple norm of $\boldsymbol{x}_{h}$. First, using the stability bound given in (3.13) and the Poincaré inequality we get

$$
\begin{aligned}
\left\|\boldsymbol{x}_{h}\right\| & \leq \sigma^{\frac{1}{2}}\left\|\boldsymbol{x}_{h}\right\|_{0, \Omega}+\mu^{\frac{1}{2}}\left\|\nabla \boldsymbol{x}_{h}\right\|_{0, \Omega}+\left|\boldsymbol{x}_{h}\right|_{S} \\
& \leq C\left(\sigma^{\frac{1}{2}}+\mu^{\frac{1}{2}}\right)\left\|\pi_{h} p-p_{h}\right\|_{0, \Omega}+\left|\boldsymbol{x}_{h}\right|_{S} .
\end{aligned}
$$

Finally, using the inverse inequality (3.2), the local trace result (3.1), and the definition of the $|\cdot|_{S}$-seminorm, we get

$$
\left|\boldsymbol{x}_{h}\right|_{S} \leq C\left(1+h^{\frac{1}{2}}+\mu \tau^{\frac{1}{2}} h^{-2}\right)\left\|\pi_{h} p-p_{h}\right\|_{0, \Omega},
$$

where the constant $C$ depends on $\sigma$ and different norms of $\boldsymbol{\beta}$, but not on $\mu$. Inserting (3.15) and (3.16) into (3.14), and using that $\mu \tau^{\frac{1}{2}} h^{-2} \leq C \mu^{\frac{1}{2}}$, regardless of the value of $\mu$, we have proven the following error estimate for the discrete pressure.

TheOREm 8. Let us assume the hypotheses of Theorem 6. Then, there exists $C>0$, independent of $h$ and $\mu$, such that

$$
\left\|\pi_{h} p-p_{h}\right\|_{0, \Omega} \leq C\left(1+\mu^{\frac{1}{2}}+h^{\frac{1}{2}}\right)\left\|\boldsymbol{u}-\boldsymbol{u}_{h}\right\| .
$$

Remark 9. The last result states that the difference $\pi_{h} p-p_{h}$ satisfies the same error estimate as the velocity, independently of the value of $\mu$. In particular, this difference behaves like $O\left(h^{k+\frac{1}{2}}\right)$ in the convection dominated regime. In addition, using the triangle inequality we get

$$
\left\|p-p_{h}\right\|_{0, \Omega} \leq\left\|p-\pi_{h} p\right\|_{0, \Omega}+\left\|\pi_{h} p-p_{h}\right\|_{0, \Omega} .
$$

This, combined with the bound proven in Theorem 8 and the standard approximation properties of $\pi_{h}$ (see, e.g., [34]), gives an optimal order $O\left(h^{k}\right)$ error estimate for the pressure whenever $Q_{h}$ contains piecewise polynomials of order $k-1$ (the case of, e.g., Scott-Vogelius elements of order $k$ ), and the pressure $p$ is regular enough. However, due to the degree of the polynomials belonging to $Q_{h}$, this error bound cannot be improved. 
4. Numerical examples. This section illustrates the theoretical findings with several numerical examples and compares the SUPG method with the new LSVS applied to the Scott-Vogelius finite element method of order 2, given by

$$
\boldsymbol{V}_{h}=\boldsymbol{W}_{h}^{2} \quad \text { and } \quad Q_{h}:=\left\{q_{h} \in L_{0}^{2}(\Omega):\left.q_{h}\right|_{K} \in \mathbb{P}_{1}(K) \forall K \in \mathcal{T}_{h}\right\} .
$$

Inf-sup stability is ensured on barycentric refined triangulations as the ones used in the examples below. The detailed implementation is stated below and all computations were performed using the finite element package ParMooN [62] and are compared and confirmed with a code written using FENiCS [50].

The discrete problem reads, Find $\left(\boldsymbol{u}_{h}, p_{h}\right) \in \boldsymbol{V}_{h} \times Q_{h}$ such that, for all $\left(\boldsymbol{v}_{h}, q_{h}\right) \in$ $V_{h} \times Q_{h}$, the following holds:

$$
a\left(\boldsymbol{u}_{h}, \boldsymbol{v}_{h}\right)+b\left(p_{h}, \boldsymbol{v}_{h}\right)+b\left(q_{h}, \boldsymbol{u}_{h}\right)+S_{\text {stab }}\left(\boldsymbol{u}_{h}, \boldsymbol{v}_{h}\right)=L_{\text {stab }}\left(\boldsymbol{v}_{h}\right),
$$

where $S_{\text {stab }}$ and $L_{\text {stab }}$ can be either the novel LSVS stabilization given by (2.9) an (2.11) or the SUPG stabilization given by

$$
\begin{aligned}
S_{\mathrm{SUPG}}\left(\boldsymbol{u}_{h}, \boldsymbol{v}_{h}\right) & :=\delta_{0} \sum_{K \in \mathcal{T}_{h}} h_{K}^{2}\left(\mathcal{L} \boldsymbol{u}_{h}, \boldsymbol{\beta} \cdot \nabla \boldsymbol{v}_{h}\right)_{K}, \\
L_{\mathrm{SUPG}}\left(\boldsymbol{v}_{h}\right) & :=\left(\boldsymbol{f}, \boldsymbol{v}_{h}\right)_{\Omega}+\delta_{0} \sum_{K \in \mathcal{T}_{h}} h_{K}^{2}\left(\boldsymbol{f}, \boldsymbol{\beta} \cdot \nabla \boldsymbol{v}_{h}\right)_{K} .
\end{aligned}
$$

To assess the influence of the stabilization parameter $\delta_{0}$ in SUPG and LSVS methods, the positive constant $\delta_{0}$ varies across the wide range from $10^{-5}$ to $10^{3}$. Concerning the choice of stabilization parameter for convection-dominated problems, e.g., see [4], a good parameter choice for the SUPG method is $\delta_{0} \in(0,1)$. Based on a parameter study presented in the next section, and from previous experience (see, e.g., [1, 4]), all the simulations for convergence studies were performed with $\delta_{0}=0.25$ for the SUPG method and $\delta_{0}=0.006$ for the LSVS method. Additionally, Example 1, Figure 5, confirms that the present method presents a much more robust behavior with respect to the value of $\delta_{0}$ than the SV-SUPG method.

4.1. Numerical results. We visit four different examples of the steady-state Oseen problem defined on the domain $\Omega=(0,1)^{2}$. All calculations are carried out on nonuniform grids. Thus, a sequence of shape-regular unstructured grids was generated, and each of these grids was barycentrically refined, thereafter, in order to guarantee inf-sup stability. The coarsest grid is depicted in Figure 1. The corresponding velocity and pressure degrees of freedom are listed next to it. In all the tables below, we use the following shorthand notation:

$$
L^{2}(u):=\left\|\boldsymbol{u}-\boldsymbol{u}_{h}\right\|_{0, \Omega}, \quad H^{1}(u):=\left\|\nabla\left(\boldsymbol{u}-\boldsymbol{u}_{h}\right)\right\|_{0, \Omega}, \quad L^{2}(p):=\left\|p-p_{h}\right\|_{0, \Omega} .
$$

4.1.1. Example 1: Potential flow example. The first example concerns a steady potential flow of the form $\boldsymbol{u}=\nabla h$ with harmonic potential $h=x^{3}-3 x y^{2}$. Then, the solution

$$
(\boldsymbol{u}, p)=\left(\nabla h,-\frac{1}{2}|\nabla h|^{2}+\frac{14}{5}\right)
$$

satisfies the Oseen problem (1.9) with the source term $\boldsymbol{f}=\mathbf{0}, \boldsymbol{\beta}=\boldsymbol{u}$, and inhomogeneous Dirichlet boundary conditions. 


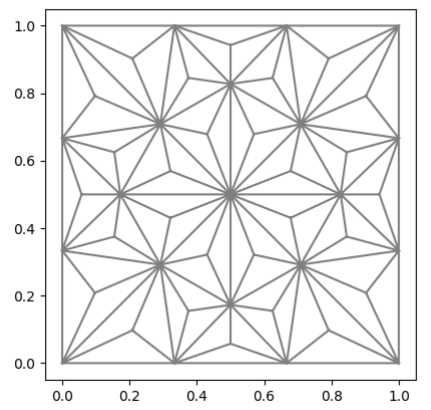

\begin{tabular}{l|lll} 
level & ndof $\boldsymbol{u}_{h}$ & ndof $p_{h}$ & total ndof \\
\hline 1 & 362 & 252 & 614 \\
2 & 1394 & 1008 & 2402 \\
3 & 5474 & 4032 & 9506 \\
4 & 21698 & 16128 & 37826 \\
5 & 86402 & 64512 & 150914
\end{tabular}

FIG. 1. Initial mesh level 1 (left) and number of degrees of freedom for all refinement levels (right).
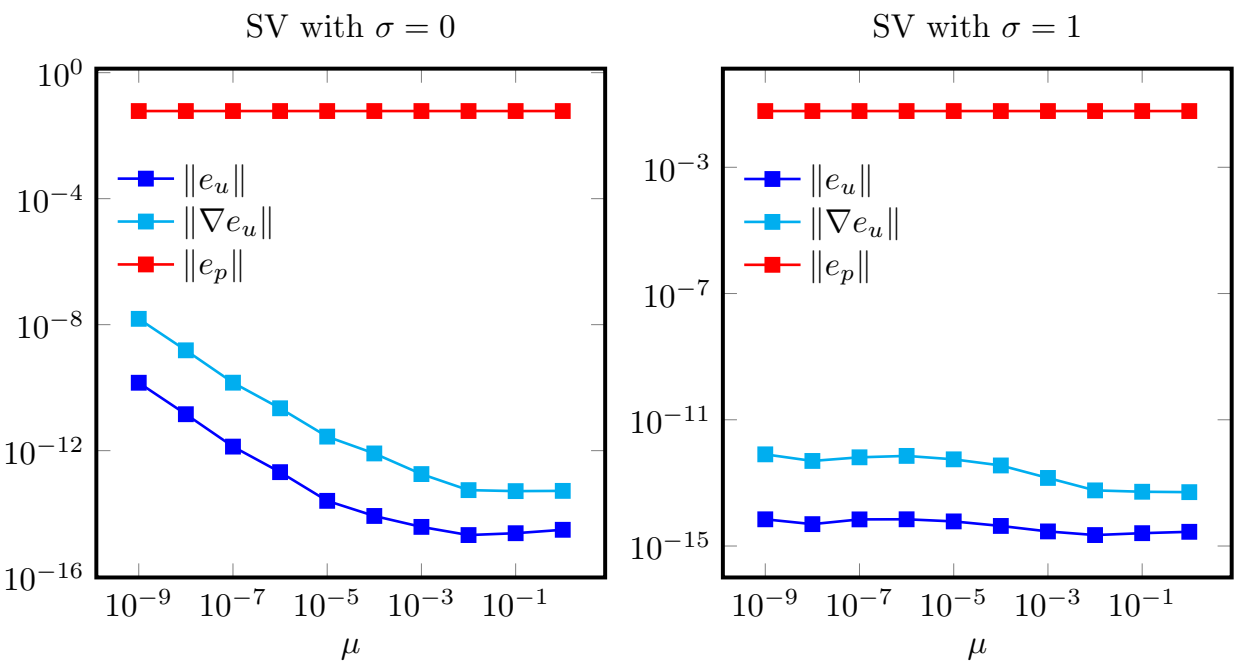

FIG. 2. Example 1: error plots of different norms versus the viscosity parameter $\mu$ for ScottVogelius finite element methods on refinement level $2(\sigma=0$ left and $\sigma=1$ right).

Figures 2, 3, and 4 display the results obtained by the plain divergence-free Galerkin Scott-Vogelius finite element method (SV), the novel least squares vorticity convection stabilization (SV-LSVS), method and the classical streamline-upwind Petrov-Galerkin (SV-SUPG) method, respctively, on refinement level 2 and the two parameter choices $\sigma=0$ and $\sigma=1$.

The main observation is that both the plain SV method and the SV-LSVS method produce the exact velocity solution in this example, while the SV-SUPG method does not. Note that this example is designed such that the exact solution belongs to the velocity ansatz space and any pressure-robust method therefore should be able to compute it exactly. For Example 4 (the most general one where the convective term has a divergence-free and an irrotational part), SV-LSVS does not present a preasymptotic regime, that was present for both plain SV and SV-SUPG. Moreover, at least in the parameter range $\mu \in\left[10^{-4}, 10^{0}\right]$ the velocity error scales with $\mu^{-1}$, which hints at a locking effect as observed for classical non-pressure-robust finite element 

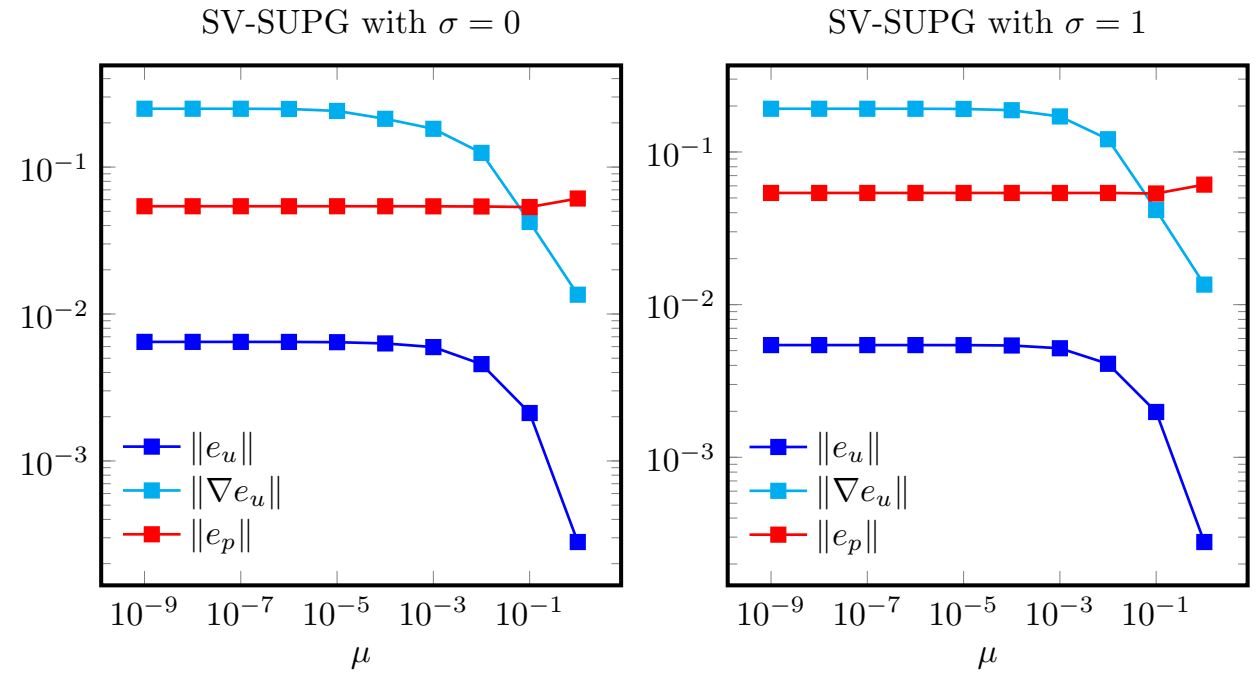

FIG. 3. Example 1: error plots of different norms versus the viscosity parameter $\mu$ for ScottVogelius element with SUPG stabilization on refinement level 2 ( $\sigma=0$ left and $\sigma=1$ right).
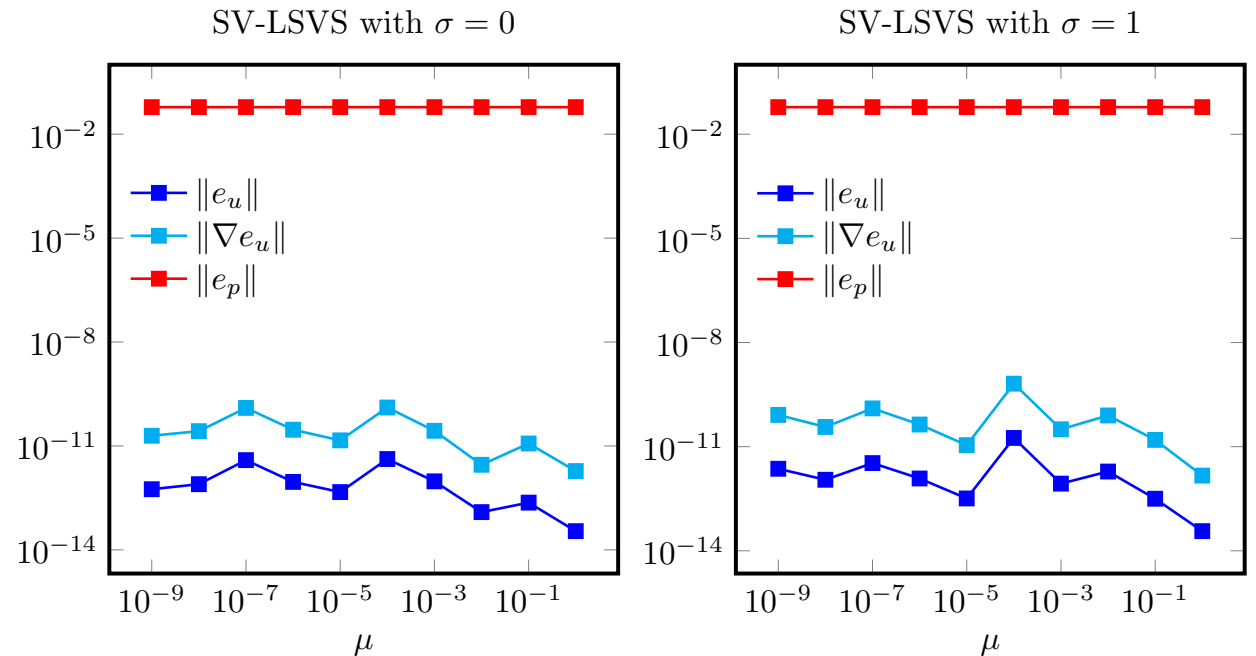

FIG. 4. Example 1: error plots of different norms versus the viscosity parameter $\mu$ for ScottVogelius finite element method with LSVS stabilization on refinement level 2 ( $\sigma=0$ left and $\sigma=1$ right).

methods in pressure-dominant situations. The effects can be explained by a closer look at the convection term. In this example $\sigma \boldsymbol{u}+(\boldsymbol{\beta} \cdot \nabla) \boldsymbol{u}$ completely balances the pressure gradient and therefore is a gradient itself. A pressure-robust stabilization does not need to stabilize gradient forces and therefore SV-LSVS (since any curl of a gradient vanishes) does not see this gradient and behaves identically to the plain SV method here - independent of the choice of the stabilization parameter. The SV-SUPG method on the other hand effectively sees and tries to stabilize the force $\nabla_{h}\left(p-p_{h}\right)$ which does not vanish. 
SV-SUPG with $\sigma=0$

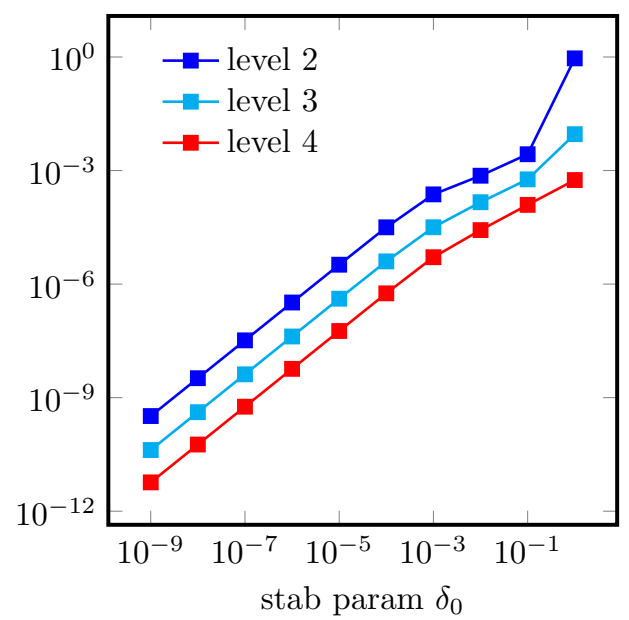

SV-SUPG with $\sigma=1$

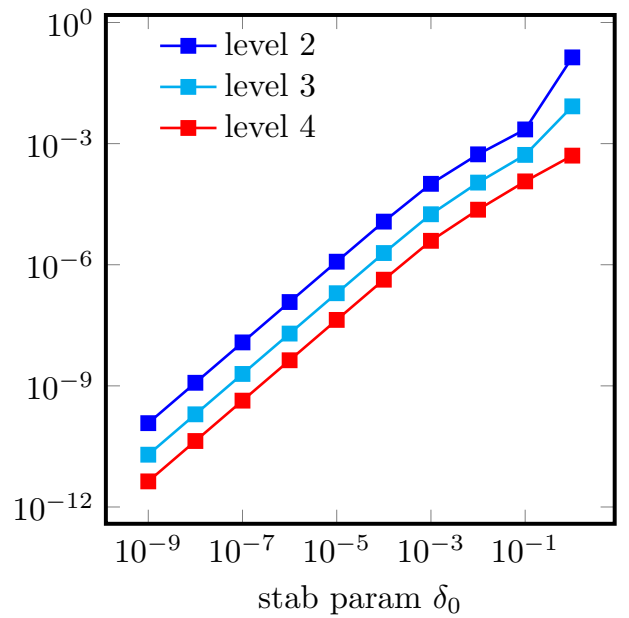

FIG. 5. Example 1: $L^{2}$ velocity error for different stabilization parameters and different refinement levels for $S V$-SUPG ( $\sigma=0$ left and $\sigma=1$ right) and fixed viscosity $\mu=10^{-5}$.

To round up the impression, Figure 5 displays the $L^{2}$ velocity error of the SVSUPG method on different mesh refinement levels and different choices of the SUPG stabilization parameter $\delta_{0}$. Usually, such a parameter plot leads to the conclusion that the optimal choice of $\delta_{0}$ is around 0.25 . This is not the case in this extreme example. Here, the error scales approximately linearly with $\delta_{0}$ and is optimal for $\delta_{0}=0$, thus reinforcing the idea that the SUPG stabilization introduces a consistency error that affects the accuracy of the method.

4.2. Example 2: Planar lattice flow. In this example, we compare the accuracy of all methods considered in the previous example. This time the exact velocity is not in the velocity ansatz space. However, the convection term is still a gradient in the limit $\boldsymbol{u}_{h} \rightarrow \boldsymbol{u}$. To this end, we fix $\mu=10^{-5}, \boldsymbol{\beta}=\boldsymbol{u}$ and boundary conditions are chosen such that

$$
\boldsymbol{u}=(\sin (2 \pi x) \sin (2 \pi y), \cos (2 \pi x) \cos (2 \pi y)), \quad p=\frac{1}{4}(\cos (4 \pi x)-\cos (4 \pi y))
$$

is the solution of the Oseen problem (1.9) with $\boldsymbol{f}=\sigma \boldsymbol{u}-\mu \Delta \boldsymbol{u}$.

Figures 6-8 display the convergence history of all three methods under consideration. The plain SV method does not converge optimally, at least pre-asymptotically for $\sigma=1$ (average $\mathrm{EOC}=2.35$ ). Also the SV-SUPG method shows suboptimal behavior for $\sigma=1$ (average $\mathrm{EOC}=2.24$ ) and for $\sigma=0$ (average $\mathrm{EOC}=1.95$ ). SV-SUPG is not really much more accurate than the plain SV method on finer meshes, while it stabilizes the solution on coarser meshes. Also, for other choices of the SUPG stabilization parameter $\delta_{0}$ (see Figure 9), the situation does not improve much, although the optimum on coarse meshes seems to be slightly shifted toward smaller values.

The SV-LSVS method on the other hand shows optimal convergence rates for $\sigma=1$ (average $\mathrm{EOC}=2.96$ ) and delivers much smaller velocity errors on the finest mesh than the other two methods; compare also the numbers in Tables 1 and 2 for $\sigma=0$ and $\sigma=1$, respectively. Figure 10 shows a similar parameter study for 

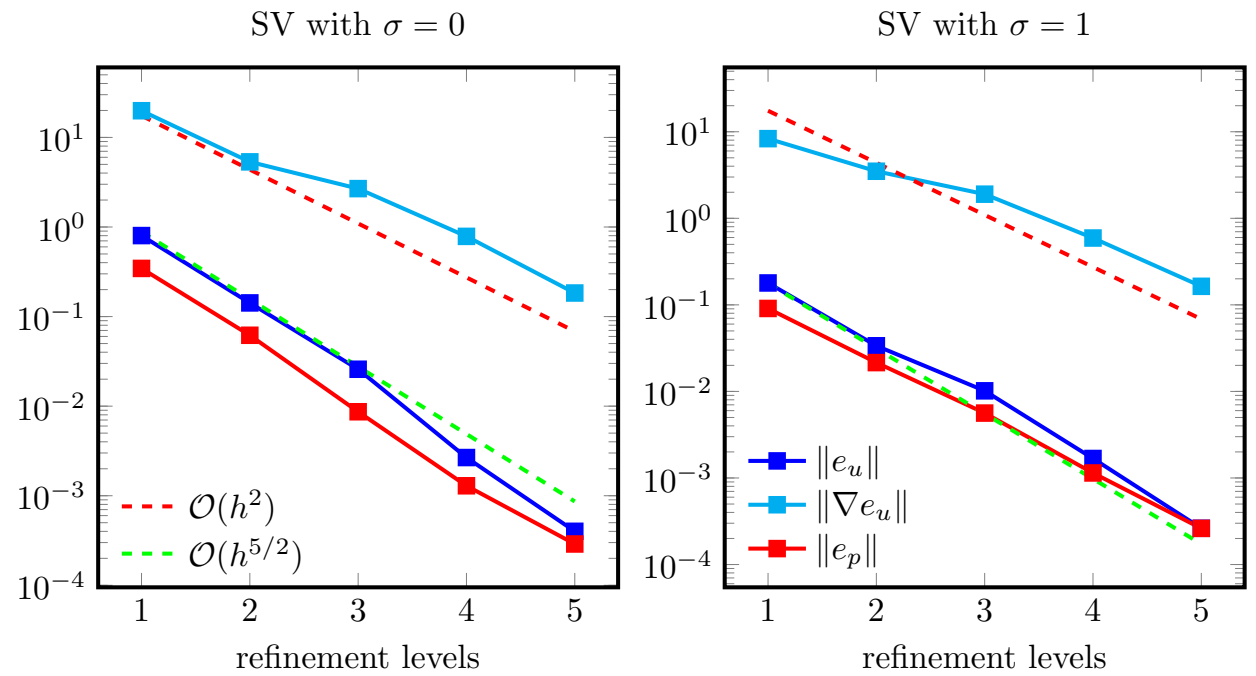

FIG. 6. Example 2: error plots of different norms on different refinement levels for ScottVogelius finite element methods ( $\sigma=0$ left and $\sigma=1$ right) and fixed viscosity $\mu=10^{-5}$.
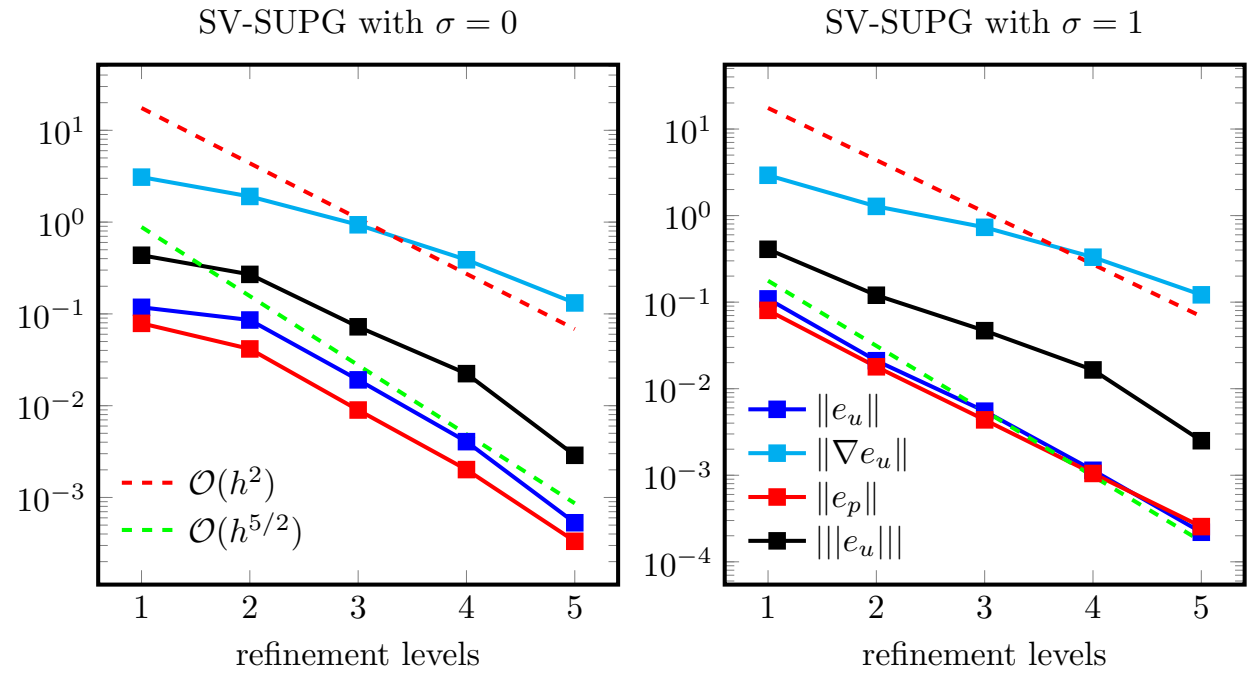

FIG. 7. Example 2: error plots of different norms on different refinement levels for ScottVogelius with SUPG stabilization ( $\sigma=0$ left and $\sigma=1$ right) and fixed viscosity $\mu=10^{-5}$.

SV-LSVS. One can see for both $\sigma=0$ and $\sigma=1$ that the optimal value lies between the interval $10^{-2}$ to $10^{-3}$.

4.3. Example 3: Modified Planar lattice flow. The third example takes the flow $\boldsymbol{u}$ of Example 2 and modifies the right-hand side forcing such that $\boldsymbol{\beta}=(0,1)^{T}$ and $p=0$.Note that this time $(\boldsymbol{\beta} \cdot \nabla) \boldsymbol{u}$ is a divergence-free field. Therefore, it is expected that this example defines the best-case scenario for the SV-SUPG method due to $p=0$. In fact, this is the case, as SV-SUPG does improve the results given by the plain Galerkin method, but still SV-LSVS provides a more accurate solution. 

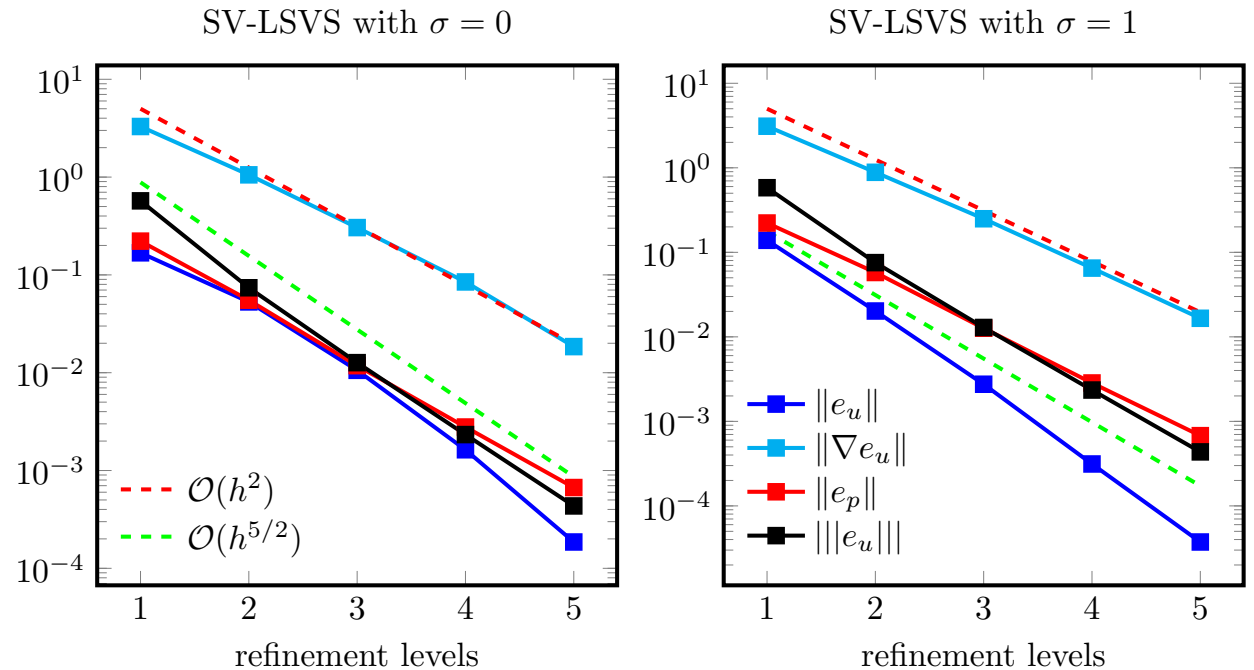

FIG. 8. Example 2: error plots of different norms on different refinement levels for ScottVogelius with LSVS stabilization ( $\sigma=0$ left and $\sigma=1$ right) and fixed viscosity $\mu=10^{-5}$.
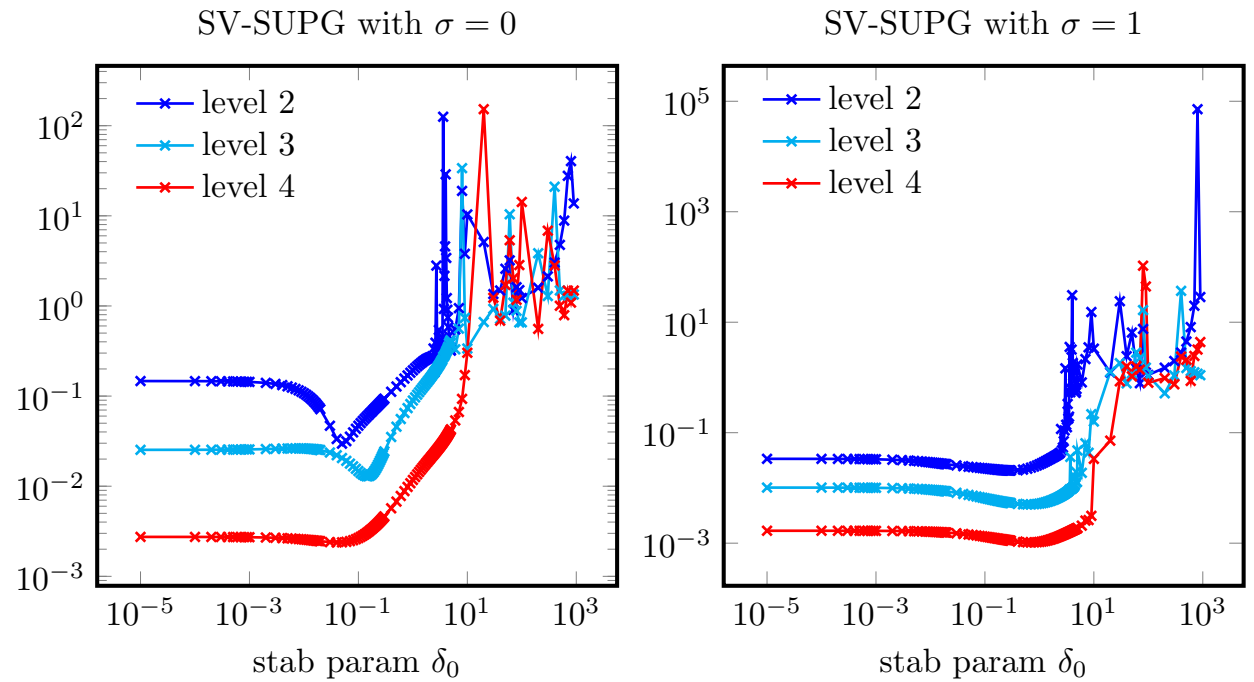

FIG. 9. Example 2: $L^{2}$ velocity error for different stabilization parameters and different refinement levels for $S V$-SUPG $\left(\sigma=0\right.$ left and $\sigma=1$ right) and fixed viscosity $\mu=10^{-5}$.

TABLE 1

Example 2: velocity and pressure errors for all methods and different refinement levels for $\sigma=0$.

\begin{tabular}{l|lll|lll|lll|}
\hline ref & \multicolumn{3}{|c|}{ SV } & \multicolumn{3}{c|}{ SV-SUPG } & \multicolumn{3}{c}{ SV-LSVS } \\
& $L^{2}(u)$ & $H^{1}(u)$ & $L^{2}(p)$ & $L^{2}(u)$ & $H^{1}(u)$ & $L^{2}(p)$ & $L^{2}(u)$ & $H^{1}(u)$ & $L^{2}(p)$ \\
\hline 1 & $8.020 \mathrm{e}-1$ & 19.86 & $3.448 \mathrm{e}-1$ & $1.179 \mathrm{e}-1$ & 3.090 & $7.888 \mathrm{e}-2$ & $1.681 \mathrm{e}-1$ & 3.2900 & $2.213 \mathrm{e}-1$ \\
2 & $1.420 \mathrm{e}-1$ & 5.335 & $6.186 \mathrm{e}-2$ & $8.578 \mathrm{e}-2$ & 1.903 & $4.152 \mathrm{e}-2$ & $5.295 \mathrm{e}-2$ & 1.0544 & $5.514 \mathrm{e}-2$ \\
3 & $2.582 \mathrm{e}-2$ & 2.682 & $8.659 \mathrm{e}-3$ & $1.911 \mathrm{e}-2$ & $9.348 \mathrm{e}-1$ & $8.968 \mathrm{e}-3$ & $1.058 \mathrm{e}-2$ & $3.045 \mathrm{e}-1$ & $1.180 \mathrm{e}-2$ \\
4 & $2.668 \mathrm{e}-3$ & $7.860 \mathrm{e}-1$ & $1.291 \mathrm{e}-3$ & $4.056 \mathrm{e}-3$ & $3.888 \mathrm{e}-1$ & $2.012 \mathrm{e}-3$ & $1.629 \mathrm{e}-3$ & $8.472 \mathrm{e}-2$ & $2.784 \mathrm{e}-3$ \\
5 & $4.007 \mathrm{e}-4$ & $1.832 \mathrm{e}-1$ & $2.891 \mathrm{e}-4$ & $5.303 \mathrm{e}-4$ & $1.316 \mathrm{e}-1$ & $3.333 \mathrm{e}-4$ & $1.858 \mathrm{e}-4$ & $1.848 \mathrm{e}-2$ & $6.697 \mathrm{e}-4$ \\
\hline EOC & 2.74 & 1.69 & 2.55 & 1.95 & 1.14 & 1.97 & 2.46 & 1.87 & 2.09 \\
\hline
\end{tabular}

Copyright (c) by SIAM. Unauthorized reproduction of this article is prohibited. 
TABLE 2

Example 2: velocity and pressure errors for all methods and different refinement levels for $\sigma=1$.

\begin{tabular}{l|lll|lll|lll|}
\hline ref & \multicolumn{3}{|c|}{ SV } & \multicolumn{3}{c|}{ SV-SUPG } & \multicolumn{3}{c}{ SV-LSVS } \\
& $L^{2}(u)$ & $H^{1}(u)$ & $L^{2}(p)$ & $L^{2}(u)$ & $H^{1}(u)$ & $L^{2}(p)$ & $L^{2}(u)$ & $H^{1}(u)$ & $L^{2}(p)$ \\
\hline 1 & $1.790 \mathrm{e}-1$ & 8.326 & $9.088 \mathrm{e}-2$ & $1.090 \mathrm{e}-1$ & 2.923 & $8.038 \mathrm{e}-2$ & $1.387 \mathrm{e}-1$ & 3.1052 & $2.222 \mathrm{e}-1$ \\
2 & $3.367 \mathrm{e}-2$ & 3.497 & $2.152 \mathrm{e}-2$ & $2.105 \mathrm{e}-2$ & 1.277 & $1.790 \mathrm{e}-2$ & $2.022 \mathrm{e}-2$ & $8.847 \mathrm{e}-1$ & $5.771 \mathrm{e}-2$ \\
3 & $1.015 \mathrm{e}-2$ & 1.900 & $5.619 \mathrm{e}-3$ & $5.501 \mathrm{e}-3$ & $7.322 \mathrm{e}-1$ & $4.364 \mathrm{e}-3$ & $2.751 \mathrm{e}-3$ & $2.496 \mathrm{e}-1$ & $1.264 \mathrm{e}-2$ \\
4 & $1.679 \mathrm{e}-3$ & $5.918 \mathrm{e}-1$ & $1.142 \mathrm{e}-3$ & $1.141 \mathrm{e}-3$ & $3.306 \mathrm{e}-1$ & $1.048 \mathrm{e}-3$ & $3.133 \mathrm{e}-4$ & $6.505 \mathrm{e}-2$ & $2.846 \mathrm{e}-3$ \\
5 & $2.623 \mathrm{e}-4$ & $1.638 \mathrm{e}-1$ & $2.616 \mathrm{e}-4$ & $2.194 \mathrm{e}-4$ & $1.215 \mathrm{e}-1$ & $2.550 \mathrm{e}-4$ & $3.741 \mathrm{e}-5$ & $1.658 \mathrm{e}-2$ & $6.775 \mathrm{e}-4$ \\
\hline EOC & 2.35 & 1.42 & 2.11 & 2.24 & 1.15 & 2.08 & 2.96 & 1.89 & 2.09 \\
\hline
\end{tabular}

SV-LSVS with $\sigma=0$

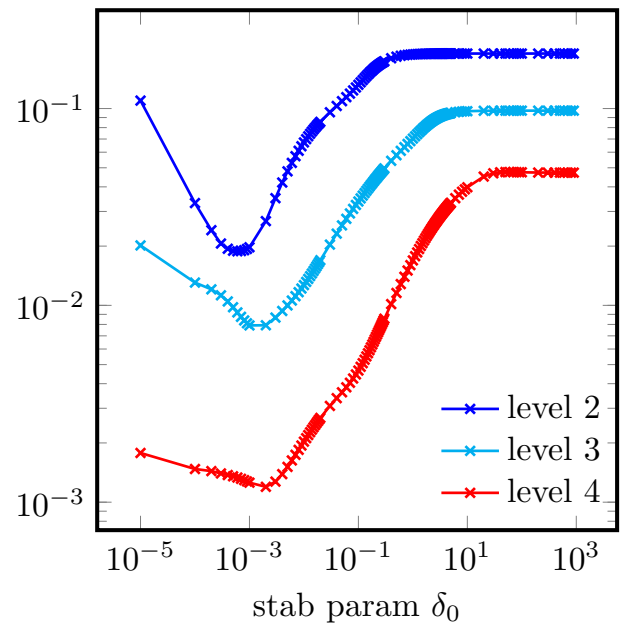

SV-LSVS with $\sigma=1$

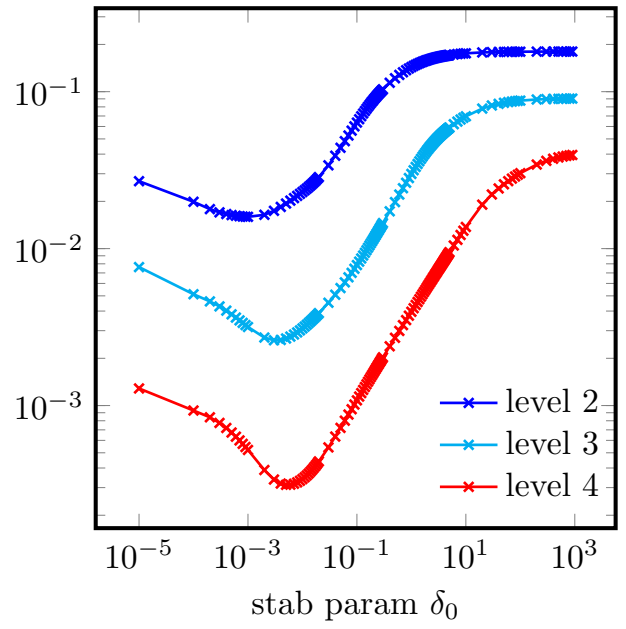

FIG. 10. Example 2: $L^{2}$ velocity error for different stabilization parameters and different refinement levels for $S V$-LSVS $\left(\sigma=0\right.$ left and $\sigma=1$ right) and fixed viscosity $\mu=10^{-5}$.

TABLE 3

Example 3: velocity and pressure errors for all methods and different refinement levels for $\sigma=0$.

\begin{tabular}{l|lll|lll|lll|}
\hline ref & \multicolumn{3}{|c|}{ SV } & \multicolumn{3}{c|}{ SV-SUPG } & \multicolumn{3}{c}{ SV-LSVS } \\
& $L^{2}(u)$ & $H^{1}(u)$ & $L^{2}(p)$ & $L^{2}(u)$ & $H^{1}(u)$ & $L^{2}(p)$ & $L^{2}(u)$ & $H^{1}(u)$ & $L^{2}(p)$ \\
\hline 1 & $4.237 \mathrm{e}-1$ & 20.605 & $2.640 \mathrm{e}-1$ & $1.672 \mathrm{e}-1$ & 4.398 & $1.207 \mathrm{e}-1$ & $1.742 \mathrm{e}-1$ & 3.1664 & $2.823 \mathrm{e}-1$ \\
2 & $7.657 \mathrm{e}-2$ & 5.6154 & $4.357 \mathrm{e}-2$ & $4.248 \mathrm{e}-2$ & 2.228 & $2.222 \mathrm{e}-2$ & $2.982 \mathrm{e}-2$ & 1.0913 & $6.163 \mathrm{e}-2$ \\
3 & $2.146 \mathrm{e}-2$ & 3.5678 & $1.323 \mathrm{e}-2$ & $9.326 \mathrm{e}-3$ & 1.041 & $5.938 \mathrm{e}-3$ & $3.875 \mathrm{e}-3$ & $3.119 \mathrm{e}-1$ & $1.320 \mathrm{e}-2$ \\
4 & $4.124 \mathrm{e}-3$ & 1.3164 & $2.561 \mathrm{e}-3$ & $1.832 \mathrm{e}-3$ & $4.462 \mathrm{e}-1$ & $1.300 \mathrm{e}-3$ & $4.836 \mathrm{e}-4$ & $7.899 \mathrm{e}-2$ & $2.307 \mathrm{e}-3$ \\
5 & $5.356 \mathrm{e}-4$ & $3.1968 \mathrm{e}-1$ & $3.835 \mathrm{e}-4$ & $3.793 \mathrm{e}-4$ & $1.818 \mathrm{e}-1$ & $2.969 \mathrm{e}-4$ & $5.916 \mathrm{e}-5$ & $1.918 \mathrm{e}-2$ & $3.389 \mathrm{e}-4$ \\
\hline EOC & 2.41 & \multicolumn{9}{c}{1.50} & 2.36 & 2.20 & 1.15 & 2.17 & 2.88 & 1.84 & 2.43 \\
\hline
\end{tabular}

TABLE 4

Example 3: velocity and pressure errors for all methods and different refinement levels for $\sigma=1$.

\begin{tabular}{l|lll|lll|lll|}
\hline ref & \multicolumn{3}{|c|}{ SV } & \multicolumn{3}{c|}{ SV-SUPG } & \multicolumn{3}{c}{ SV-LSVS } \\
& $L^{2}(u)$ & $H^{1}(u)$ & $L^{2}(p)$ & $L^{2}(u)$ & $H^{1}(u)$ & $L^{2}(p)$ & $L^{2}(u)$ & $H^{1}(u)$ & $L^{2}(p)$ \\
\hline 1 & $3.397 \mathrm{e}-1$ & 17.27 & $2.402 \mathrm{e}-1$ & $1.518 \mathrm{e}-1$ & 4.056 & $1.203 \mathrm{e}-1$ & $1.536 \mathrm{e}-1$ & 3.1088 & $2.911 \mathrm{e}-1$ \\
2 & $6.118 \mathrm{e}-2$ & 5.188 & $4.125 \mathrm{e}-2$ & $3.504 \mathrm{e}-2$ & 1.927 & $2.386 \mathrm{e}-2$ & $2.626 \mathrm{e}-2$ & 1.0425 & $6.320 \mathrm{e}-2$ \\
3 & $1.694 \mathrm{e}-2$ & 2.781 & $1.115 \mathrm{e}-2$ & $7.981 \mathrm{e}-3$ & $9.191 \mathrm{e}-1$ & $5.768 \mathrm{e}-3$ & $3.483-\mathrm{e} 3$ & $3.033 \mathrm{e}-1$ & $1.331 \mathrm{e}-2$ \\
4 & $3.107 \mathrm{e}-3$ & 1.062 & $2.152 \mathrm{e}-3$ & $1.654 \mathrm{e}-3$ & $4.122 \mathrm{e}-1$ & $1.298 \mathrm{e}-3$ & $4.308 \mathrm{e}-4$ & $7.772 \mathrm{e}-2$ & $2.310 \mathrm{e}-3$ \\
5 & $4.646 \mathrm{e}-4$ & $2.952 \mathrm{e}-1$ & $3.725 \mathrm{e}-4$ & $3.490 \mathrm{e}-4$ & $1.732 \mathrm{e}-1$ & $3.014 \mathrm{e}-4$ & $5.178 \mathrm{e}-5$ & $1.905 \mathrm{e}-2$ & $3.390 \mathrm{e}-4$ \\
\hline EOC & 2.38 & 1.47 & 2.33 & 2.19 & 1.14 & 2.16 & 2.88 & 1.84 & 2.44 \\
\hline
\end{tabular}

Tables 3 and 4 confirm that SV-LSVS also works better than SV-SUPG in this example. While the results are comparable on coarse meshes, on finer meshes the L2 velocity errors of SV-LSVS get much smaller than those of SV-SUPG (up to a factor 6). Also the convergence histories of Figures 11, 12, and 13 show that the 

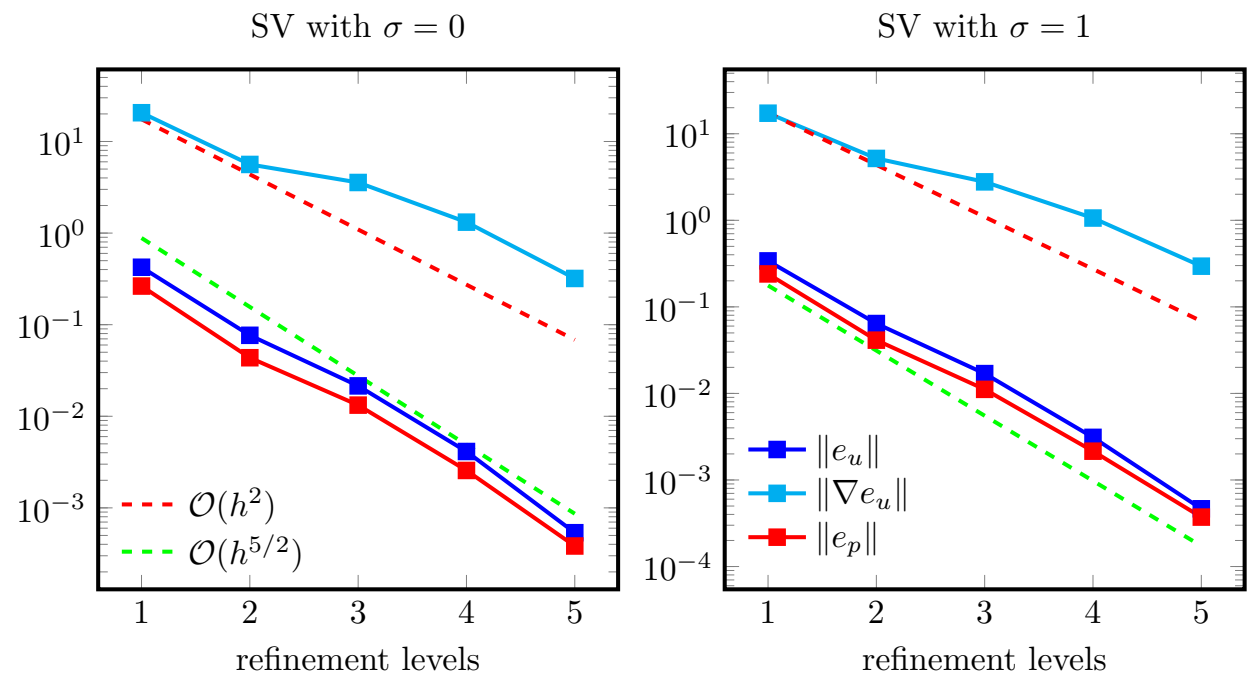

FIG. 11. Example 3: error plots of different norms on different refinement levels for ScottVogelius finite element methods ( $\sigma=0$ left and $\sigma=1$ right) and fixed viscosity $\mu=10^{-5}$.
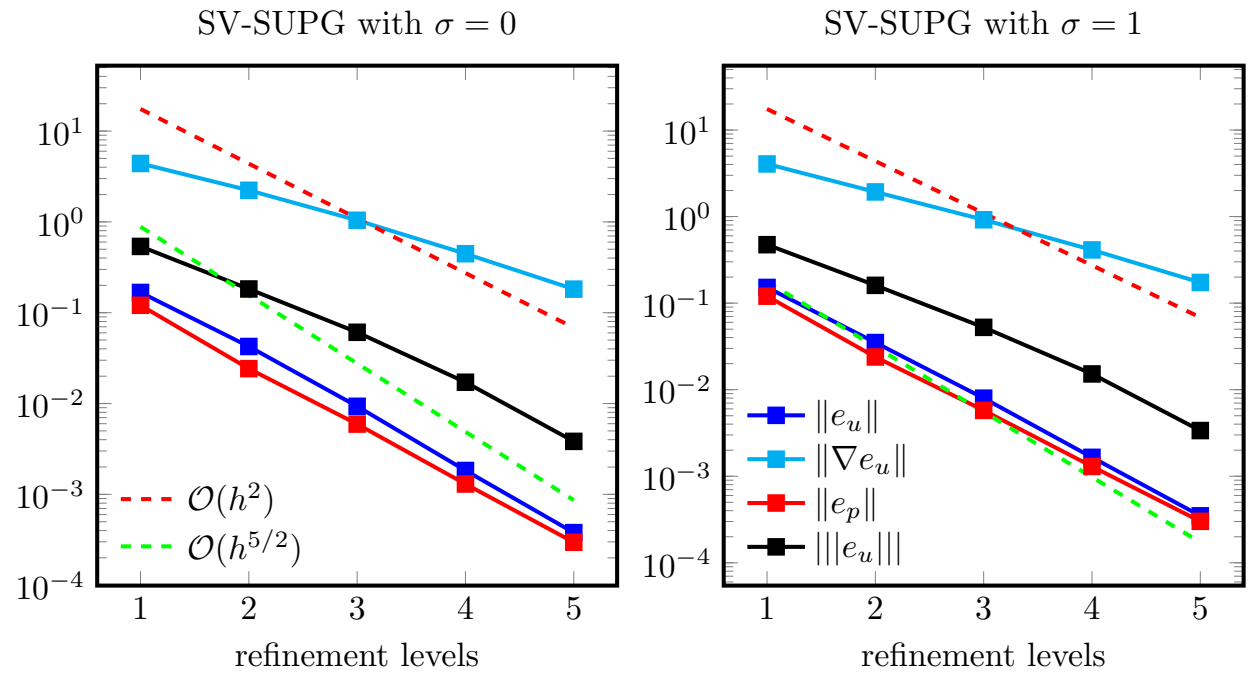

FIG. 12. Example 3: error plots of different norms on different refinement levels for ScottVogelius with SUPG stabilization ( $\sigma=0$ left and $\sigma=1$ right) and fixed viscosity $\mu=10^{-5}$.

velocity errors of SV-SUPG do not converge optimally between refinement levels 2 and 5, similar to what was observed in Example 2. Figure 14 confirms that SV-SUPG method works close to its optimum with the default parameter $\delta_{0}=0.25$. Figure 15 for the SV-LSVS method on the other hand shows that $\delta_{0}=0.006$ is a good estimate for optimal parameter value.

4.4. Example 4: "Superposition" of Examples 2 and 3. The last example combines the flows of Examples 2 and 3 and employs a superposition of their convec- 

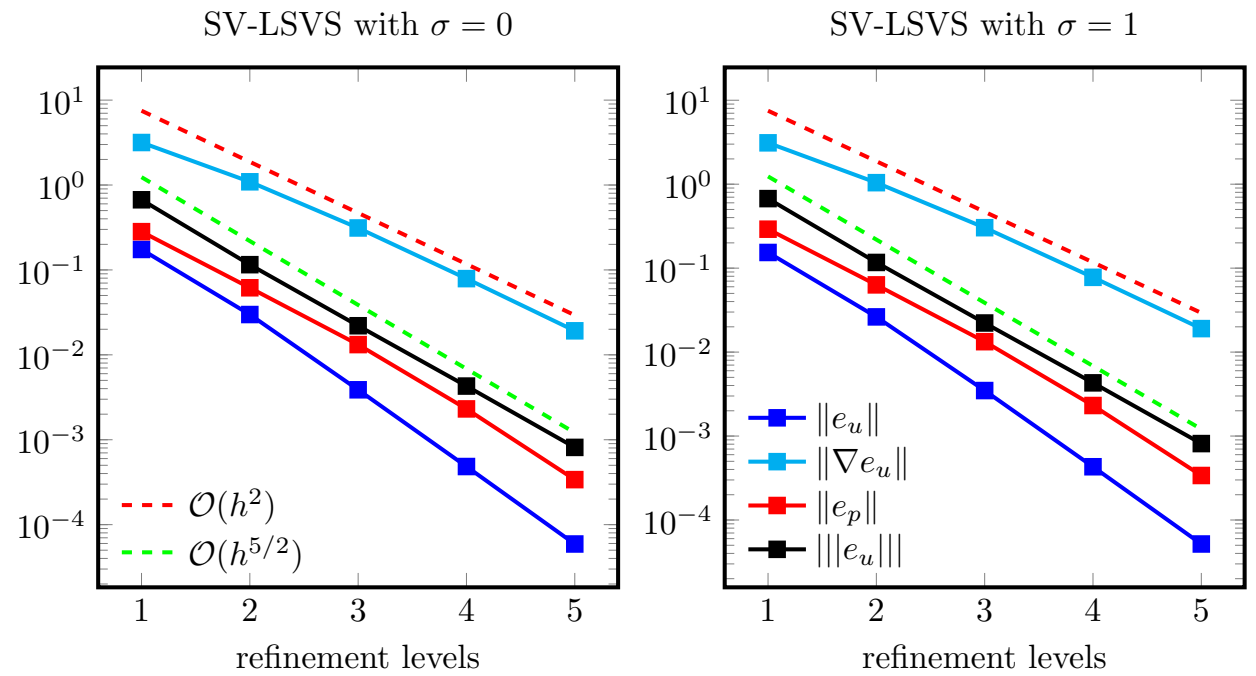

FIG. 13. Example 3: error plots of different norms on different refinement levels for ScottVogelius with LSVS stabilization ( $\sigma=0$ left and $\sigma=1$ right) and fixed viscosity $\mu=10^{-5}$.
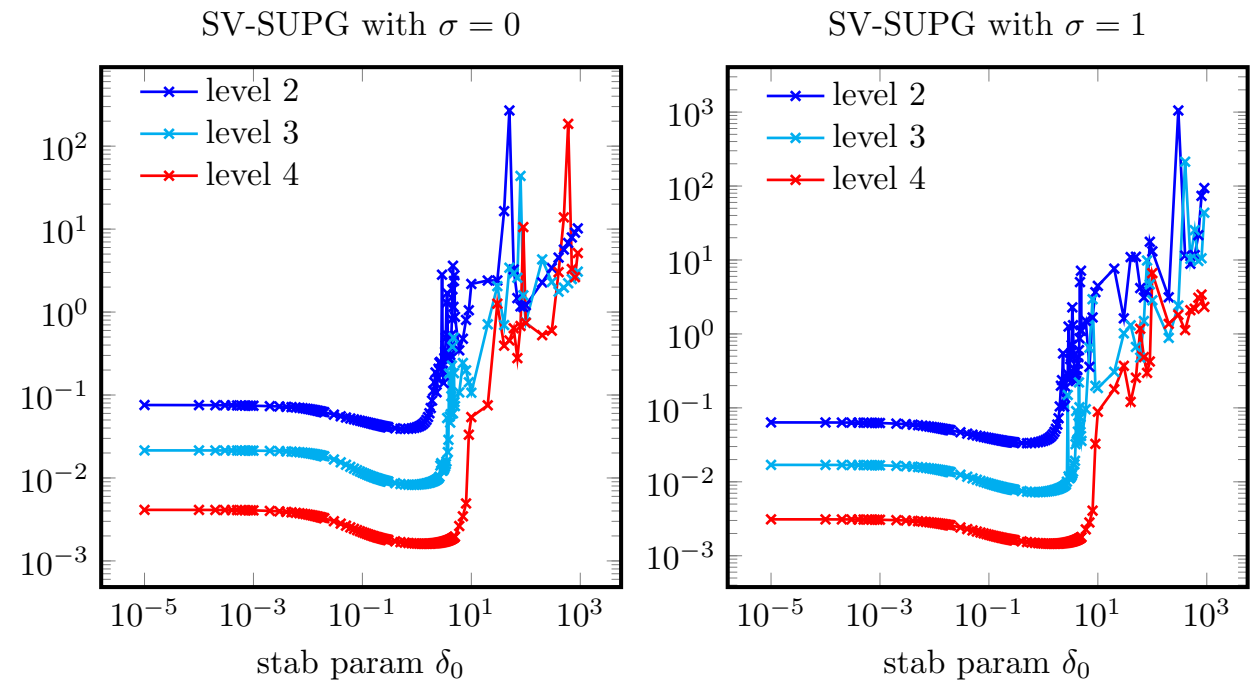

FIG. 14. Example 3: $L^{2}$ velocity error for different stabilization parameters and different refinement levels for $S V$-SUPG ( $\sigma=0$ left and $\sigma=1$ right) and fixed viscosity $\mu=10^{-5}$.

tive forces. That is, the convective term is given by $\boldsymbol{\beta}:=\boldsymbol{u}+(0,1)^{T}$, while $\boldsymbol{u}$ and $p$ are the same as in Example 2. This is somehow considered to be a "realistic" situation where the (discrete and asymptotic) convective forcing has an irrotational part (as in Examples 1 and 2) and a divergence-free part (as in Example 3).

As expected from the experience with the other examples, both stabilization methods significantly improve the errors compared to the plain SV method. There is also a clear improvement of SV-LSVS compared to SV-SUPG. Only SV-LSVS has an optimal convergence behavior; compare Figures 16-18 and Tables 5 and 6. 

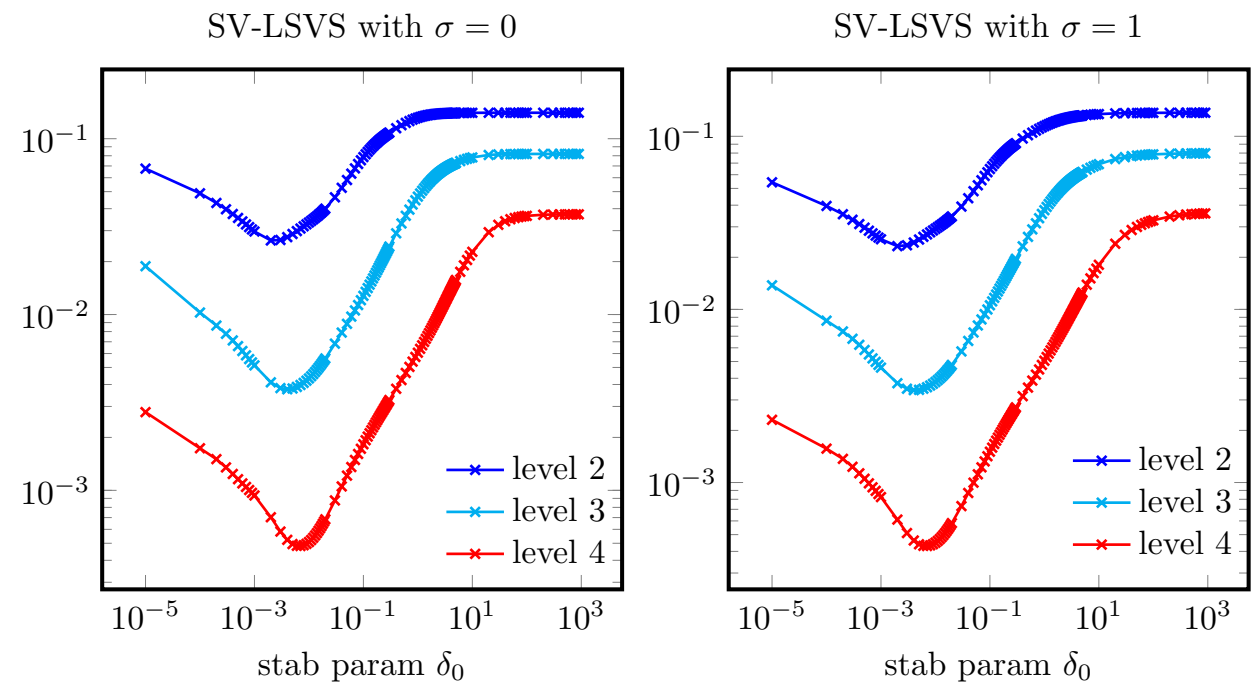

FIG. 15. Example 3: $L^{2}$ velocity error for different stabilization parameters and different refinement levels for $S V$-LSVS $\left(\sigma=0\right.$ left and $\sigma=1$ right) and fixed viscosity $\mu=10^{-5}$.
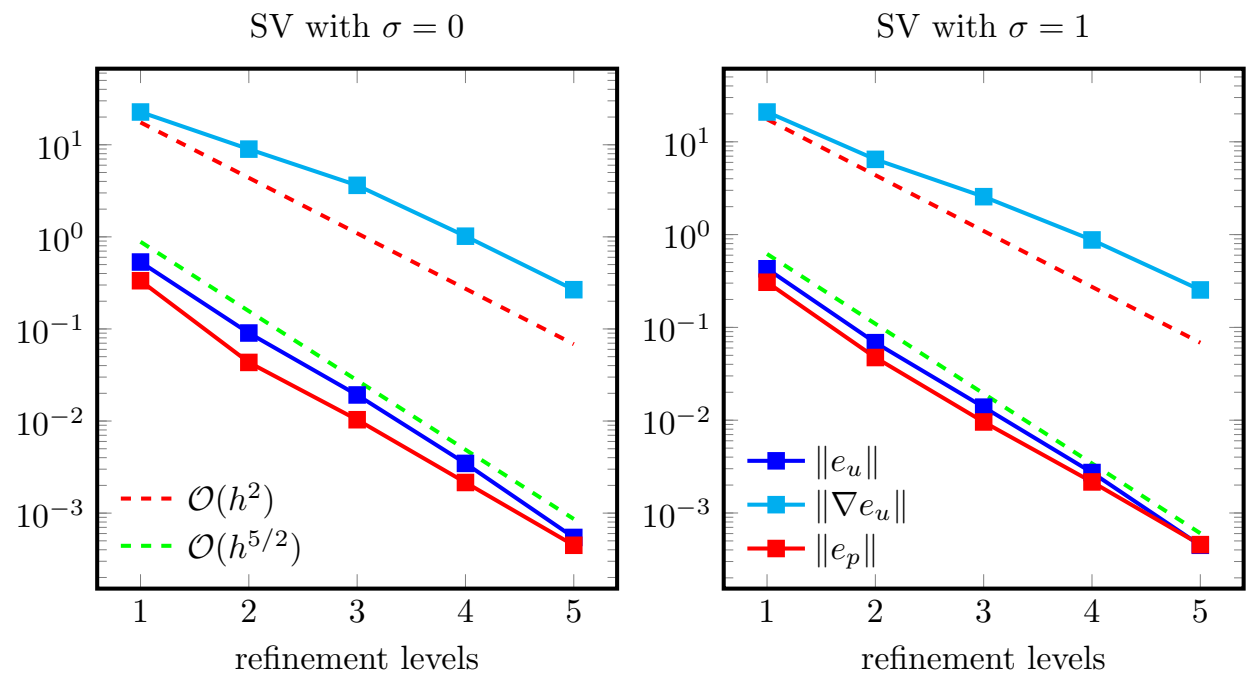

FIG. 16. Example 4: error plots of different norms on different refinement levels for ScottVogelius finite element methods ( $\sigma=0$ left and $\sigma=1$ right) and fixed viscosity $\mu=10^{-5}$.

5. Concluding remarks. In this work a new stabilized finite element method for the Oseen has been proposed and analyzed. The method is based on the observation that, in order to obtain pressure-robust error estimates, the stabilization term needs to be independent of the pressure. That is why the stabilizing term is built as a penalization of the vorticity equation, where the pressure gradient is not present. This design has allowed us to prove optimal, pressure-independent error estimates for the velocity, in particular, the $O\left(h^{k+\frac{1}{2}}\right)$ error bound for $\left\|\boldsymbol{u}-\boldsymbol{u}_{h}\right\|_{0, \Omega}$, not available for the Galerkin method or the SUPG method when applied to inf-sup stable discretizations, and also only available so far for $H^{1}$-conforming equal order stabilized methods 

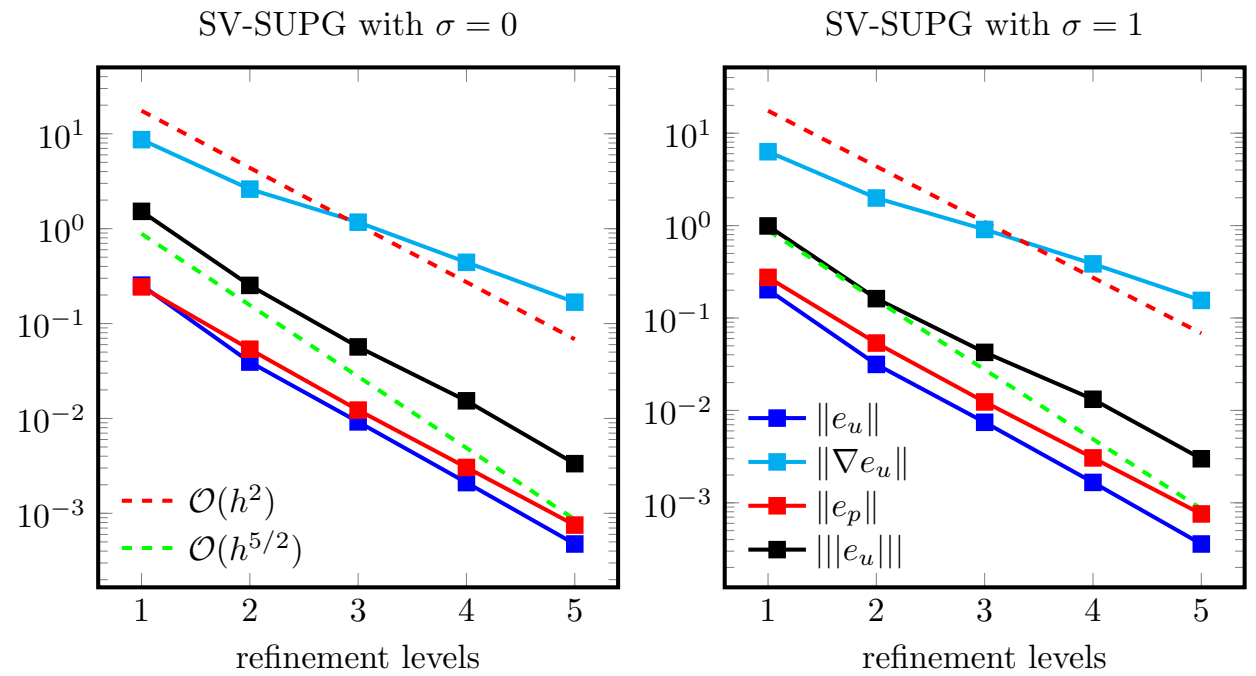

FIG. 17. Example 4: error plots of different norms on different refinement levels for ScottVogelius with SUPG stabilization ( $\sigma=0$ left and $\sigma=1$ right) and fixed viscosity $\mu=10^{-5}$.
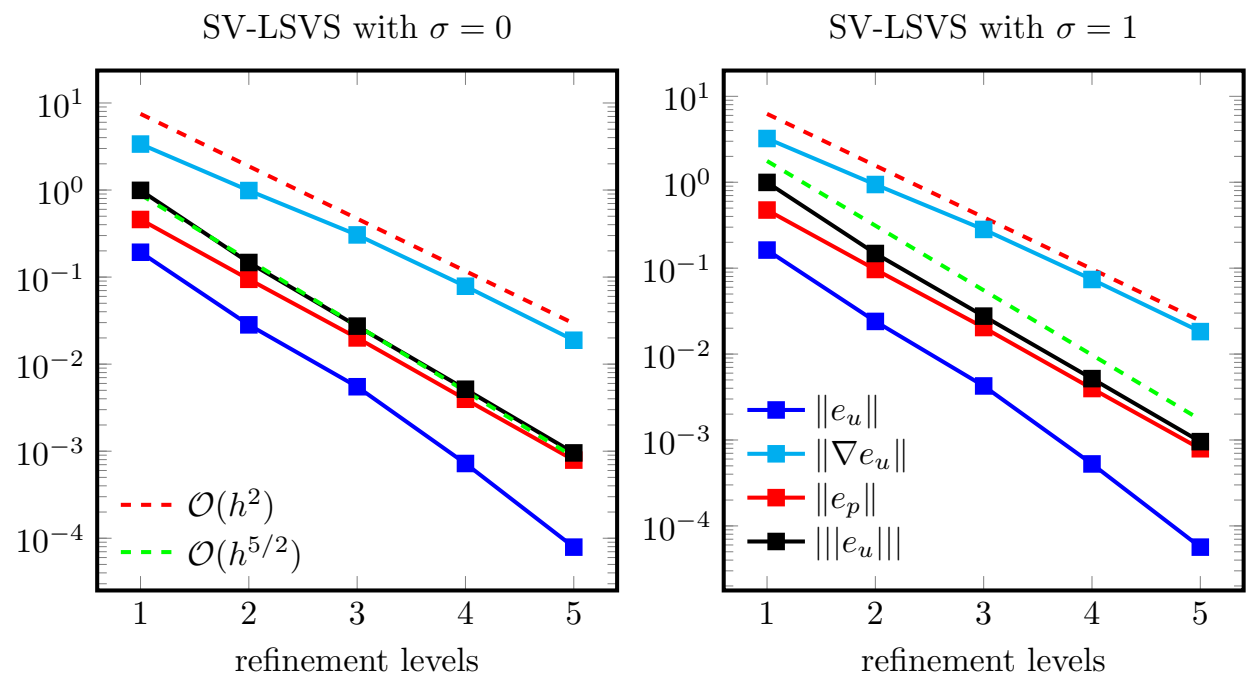

FIG. 18. Example 4: error plots of different norms on different refinement levels for ScottVogelius with convection stabilization ( $\sigma=0$ left and $\sigma=1$ right) and fixed viscosity $\mu=10^{-5}$.

(at the price of a constant that depends on the regularity of the pressure). From the numerical results we can extract the following conclusions:

- SV-LSVS works well and converges with an optimal order in any situation (Examples 1-4); in the extreme Example 1 it delivers the exact solution for every stabilization parameter.

- SV-SUPG converges always suboptimally. In situations where the convective force is close to a gradient it can be less accurate than the plain SV method. However, for situations where the convective term is divergence-free, SV-SUPG delivers more accurate results on coarse meshes than plain SV. 

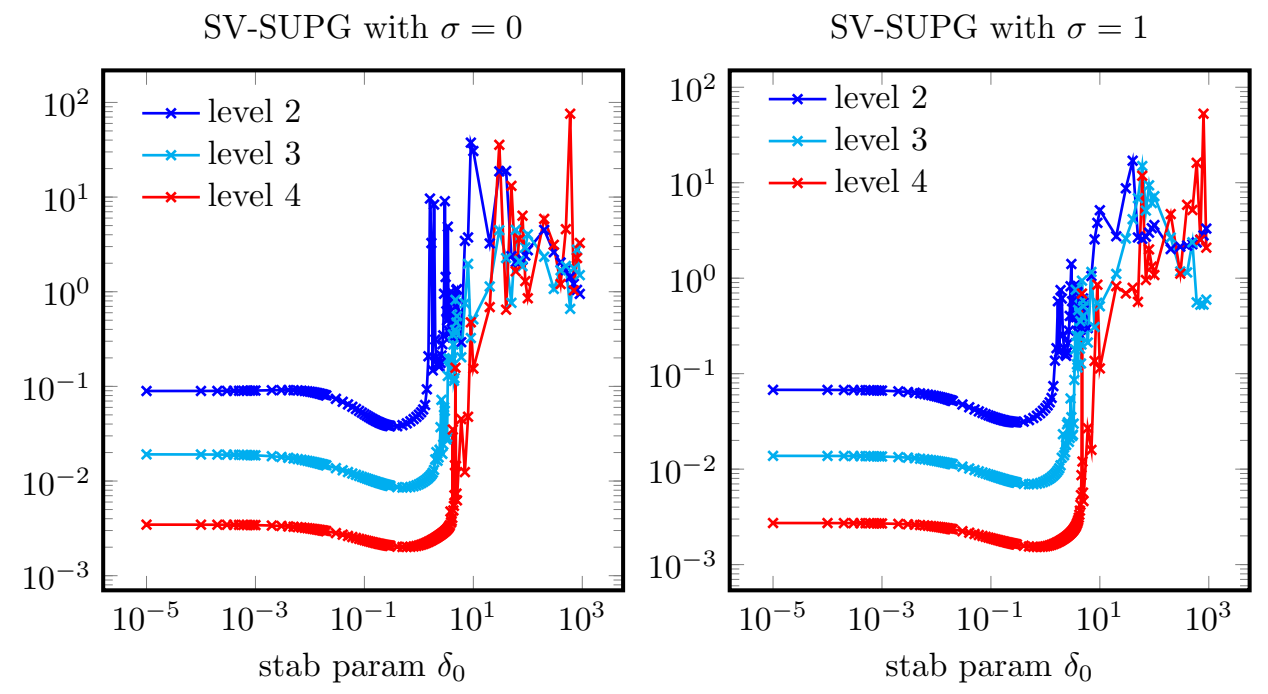

FIG. 19. Example 4: $L^{2}$ velocity error for different stabilization parameters and different refinement levels for $S V$-SUPG $\left(\sigma=0\right.$ left and $\sigma=1$ right) and fixed viscosity $\mu=10^{-5}$.
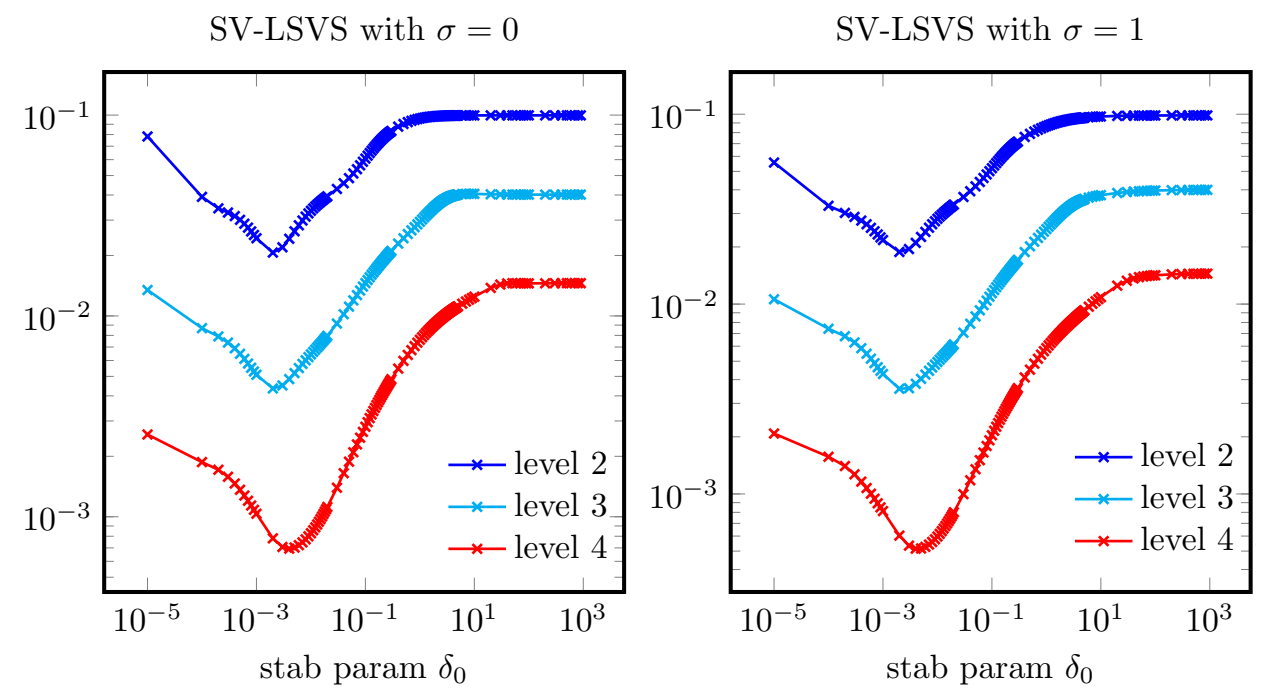

FIG. 20. Example 4: $L^{2}$ velocity error for different stabilization parameters and different refinement levels for $S V$-LSVS ( $\sigma=0$ left and $\sigma=1$ right) and fixed viscosity $\mu=10^{-5}$.

- SV-LSVS outperforms plain SV and SV-SUPG, in the most general Example 4, where the convective term has a divergence-free and an irrotational part.

- The SV-LSVS has a robust behavior with respect to the stabilization parameter. For all Examples 1-4, the same parameter $\delta_{0}=0.006$ was used. Instead, for SV-SUPG in Example 1 it could be shown that the optimal parameter is $\delta_{0}=0$, while it is about $\delta_{0} \approx 0.25$ for Examples $2-4$. 
TABLE 5

Example 4: velocity and pressure errors for all methods and different refinement levels for $\sigma=0$.

\begin{tabular}{|c|c|c|c|c|c|c|c|c|c|}
\hline \multirow[t]{2}{*}{ ref } & \multicolumn{3}{|c|}{ SV } & \multicolumn{3}{|c|}{ SV-SUPG } & \multicolumn{3}{|c|}{ SV-LSVS } \\
\hline & $L^{2}(u)$ & $H^{1}(u)$ & $L^{2}(p)$ & $L^{2}(u)$ & $H^{1}(u)$ & $L^{2}(p)$ & $L^{2}(u)$ & $H^{1}(u)$ & $L^{2}(p)$ \\
\hline 1 & $5.328 \mathrm{e}-1$ & 22.69 & $3.339 \mathrm{e}-1$ & $2.540 \mathrm{e}-1$ & 8.6706 & $2.434 \mathrm{e}-1$ & $1.929 \mathrm{e}-1$ & 3.3734 & $4.587 \mathrm{e}-1$ \\
\hline 2 & $9.032 \mathrm{e}-2$ & $8.969 \mathrm{e}+0$ & $4.330 \mathrm{e}-2$ & $3.928 \mathrm{e}-2$ & 2.6116 & $5.363 \mathrm{e}-2$ & $2.826 \mathrm{e}-2$ & $9.865 \mathrm{e}-1$ & $9.425 \mathrm{e}-2$ \\
\hline 3 & $1.919 \mathrm{e}-2$ & $3.627 \mathrm{e}+0$ & $1.033 \mathrm{e}-2$ & $9.198 \mathrm{e}-3$ & 1.1702 & $1.224 \mathrm{e}-2$ & $5.499 \mathrm{e}-3$ & $3.050 \mathrm{e}-1$ & $2.000 \mathrm{e}-2$ \\
\hline 4 & $3.467 \mathrm{e}-3$ & $1.016 \mathrm{e}+0$ & $2.150 \mathrm{e}-3$ & $2.107 \mathrm{e}-3$ & $4.421 \mathrm{e}-1$ & $3.043 \mathrm{e}-3$ & $7.221 \mathrm{e}-4$ & $7.851 \mathrm{e}-2$ & $3.949 \mathrm{e}-3$ \\
\hline 5 & $5.443 \mathrm{e}-4$ & $2.668 \mathrm{e}-1$ & $4.473 \mathrm{e}-4$ & $4.752 \mathrm{e}-4$ & $1.680 \mathrm{e}-1$ & $7.519 \mathrm{e}-4$ & $7.904 \mathrm{e}-5$ & $1.882 \mathrm{e}-2$ & $7.901 \mathrm{e}-4$ \\
\hline $\mathrm{EOC}$ & 2.48 & 1.60 & 2.39 & 2.27 & 1.42 & 2.08 & 2.81 & 1.87 & 2.30 \\
\hline
\end{tabular}

TABLE 6

Example 4: velocity and pressure errors for all methods and different refinement levels for $\sigma=1$.

\begin{tabular}{l|lll|lll|lll}
\hline ref & \multicolumn{3}{|c|}{ SV } & \multicolumn{3}{c|}{ SV-SUPG } & \multicolumn{3}{c}{ SV-LSVS } \\
& $L^{2}(u)$ & $H^{1}(u)$ & $L^{2}(p)$ & $L^{2}(u)$ & $H^{1}(u)$ & $L^{2}(p)$ & $L^{2}(u)$ & $H^{1}(u)$ & $L^{2}(p)$ \\
\hline 1 & $4.284 \mathrm{e}-1$ & 20.93 & $3.066 \mathrm{e}-1$ & $2.022 \mathrm{e}-1$ & 6.3029 & $2.751 \mathrm{e}-1$ & $1.624 \mathrm{e}-1$ & 3.2283 & $4.747-1$ \\
2 & $6.847 \mathrm{e}-2$ & $6.468 \mathrm{e}+0$ & $4.734 \mathrm{e}-2$ & $3.146 \mathrm{e}-2$ & 1.9888 & $5.358 \mathrm{e}-2$ & $2.399 \mathrm{e}-2$ & $9.408 \mathrm{e}-1$ & $9.650-2$ \\
3 & $1.382 \mathrm{e}-2$ & $2.562 \mathrm{e}+0$ & $9.569 \mathrm{e}-3$ & $7.439 \mathrm{e}-3$ & $9.080 \mathrm{e}-1$ & $1.234 \mathrm{e}-2$ & $4.255 \mathrm{e}-3$ & $2.822 \mathrm{e}-1$ & $2.036-2$ \\
4 & $2.729 \mathrm{e}-3$ & $8.759 \mathrm{e}-1$ & $2.161 \mathrm{e}-3$ & $1.663 \mathrm{e}-3$ & $3.854 \mathrm{e}-1$ & $3.062 \mathrm{e}-3$ & $5.264 \mathrm{e}-4$ & $7.382 \mathrm{e}-2$ & $3.970-3$ \\
5 & $4.487 \mathrm{e}-4$ & $2.533 \mathrm{e}-1$ & $4.561 \mathrm{e}-4$ & $3.585 \mathrm{e}-4$ & $1.550 \mathrm{e}-1$ & $7.579 \mathrm{e}-4$ & $5.662 \mathrm{e}-5$ & $1.826 \mathrm{e}-2$ & $7.907-4$ \\
\hline EOC & 2.47 & 1.59 & 2.35 & 2.28 & 1.34 & 2.13 & 2.87 & 1.87 & 2.31 \\
\hline
\end{tabular}

\section{REFERENCES}

[1] N. Ahmed And V. John, An assessment of two classes of variational multiscale methods for the simulation of incompressible turbulent flows, Comput. Methods Appl. Mech. Engrg., 365 (2020), 112997.

[2] N. Ahmed, A. Linke, And C. Merdon, On really locking-free mixed finite element methods for the transient incompressible Stokes equations, SIAM J. Numer. Anal., 56 (2018), pp. 185-209.

[3] N. Ahmed, A. Linke, And C. Merdon, Towards pressure-robust mixed methods for the incompressible Navier-Stokes equations, Comput. Methods Appl. Math., 18 (2018), pp. 353-372.

[4] N. Ahmed And G. Matthies, Numerical study of SUPG and LPS methods combined with higher order variational time discretization schemes applied to time-dependent linear convection-diffusion-reaction equations, J. Sci. Comput., 67 (2016), pp. 988-1018.

[5] V. I. Arnold And B. A. Khesin, Topological Methods in Hydrodynamics, Appl. Math. Sci. 125, Springer, New York, 1998.

[6] G. R. Barrenechea, E. Burman, and J. Guzmán, Well-posedness and H(div)-conforming finite element approximation of a linearised model for inviscid incompressible flow, Math. Models Methods Appl. Sci., 30 (2020), pp. 847-865.

[7] P. Bochev, C. R. Dohrman, and M. D. Gunzburger, Stabilisation of low-order mixed finite elements for the Stokes equations, SIAM J. Numer. Anal., 44 (2006), pp. 82-101.

[8] D. Boffi, F. Brezzi, And M. Fortin, Mixed Finite Element Methods and Applications, Springer Ser. Computat. Math. 44, Springer, New York, 2013.

[9] F. Boyer AND P. FABRIE, Mathematical Tools for the Study of the Incompressible NavierStokes Equations and Related Models, Appl. Math. Sci. 183, Springer, New York, 2013.

[10] M. BraACK AND E. Burman, Local projection stabilization for the Oseen problem and its interpretation as a variational multiscale method, SIAM J. Numer. Anal., 43 (2006), pp. $2544-2566$.

[11] M. BraAck, E. Burman, V. John, and G. Lube, Stabilized finite element methods for the generalized Oseen problem, Comput. Methods Appl. Mech. Engrg., 196 (2007), pp. 853-866.

[12] F. Brezzi and J. Pitkäranta, On the stabilization of finite element approximations of the Stokes equations, in Efficient Solutions of Elliptic Systems (Kiel, 1984), Notes Numer. Fluid Mech. 10, Friedr. Vieweg, Braunschweig, 1984, pp. 11-19.

[13] A. Buffa, C. De Falco, and G. Sangaldi, Isogeometric analysis: Stable elements for the $2 D$ Stokes equation, Int. J. Numer. Methods Fluids, 65 (2011), pp. 1407-1422.

[14] E. BuRman, Pressure projection stabilizations for Galerkin approximations of Stokes' and Darcy's problem, Numer. Methods Partial Differential Equations, 24 (2008), pp. 127-143.

[15] E. Burman, M. A. Fernández, and P. Hansbo, Continuous interior penalty finite element method for Oseen's equations, SIAM J. Numer. Anal., 44 (2006), pp. 248-1274.

[16] E. BURMAN AND P. HANSBO, Edge stabilization for Galerkin approximations of convectiondiffusion-reaction problems, Comput. Methods Appl. Mech. Engrg., 193 (2004), pp. 1437-1453, https://doi.org/10.1016/j.cma.2003.12.032. 
[17] E. Burman AND A. Linke, Stabilized finite element schemes for incompressible flow using Scott-Vogelius elements, Appl. Numer. Math., 58 (2008), pp. 1704-1719, https://doi.org/ 10.1016/j.apnum.2007.11.001.

[18] M. Case, V. Ervin, A. Linke, And L. Rebholz, A connection between Scott-Vogelius and grad-div stabilized Taylor-Hood FE approximations of the Navier-Stokes equations, SIAM J. Numer. Anal., 49 (2011), pp. 1461-1481.

[19] A. J. Chorin and J. E. Marsden, A Mathematical Introduction to Fluid Mechanics, 3rd ed., Texts in Appl. Math. 4, Springer, New York, 1993.

[20] S. H. Christiansen And K. Hu, Generalized finite element systems for smooth differential forms and Stokes' problem, Numer. Math., 140 (2018), pp. 327-371, https://doi.org/10. 1007/s00211-018-0970-6.

[21] S. H. Christiansen And K. Hu, Generalized finite element systems for smooth differential forms and Stokes' problem, Numer. Math., 140 (2018), pp. 327-371.

[22] R. W. Clough and J. L. Tocher, Finite element stiffness matrices for analysis of plates in bending, in Proceedings of the Conference on Matrix Methods in Structural Mechanics, 1965 , pp. 515-545.

[23] R. Codina, Analysis of a stabilized finite element approximation of the Oseen equations using orthogonal subscales, Appl. Numer. Math., 58 (2008), pp. 264-283.

[24] M. Costabel and A. McIntosh, On Bogovskǐ and regularized Poincaré integral operators for de Rham complexes on Lipschitz domains, Math. Z., 265 (2010), pp. 297-320.

[25] J. A. Evans AND T. J. R. Hughes, Isogeometric divergence-conforming B-splines for the steady Navier-Stokes equations, Math. Models Methods Appl. Sci., 23 (2013), pp. 1421-1478, https://doi.org/10.1142/S0218202513500139.

[26] J. A. Evans and T. J. R. Hughes, Isogeometric divergence-conforming B-splines for the unsteady Navier-Stokes equations, J. Comput. Phys., 241 (2013), pp. 141-167.

[27] R. S. Falk and M. NeIlan, Stokes complexes and the construction of stable finite elements with pointwise mass conservation, SIAM J. Numer. Anal., 51 (2013), pp. 1308-1326.

[28] N. Fehn, M. Kronbichler, C. Lehrenfeld, G. Lube, and P. W. Schroeder, High-order DG solvers for underresolved turbulent incompressible flows: $A$ comparison of $L^{2}$ and $H$ (div) methods, Internat. J. Numer. Methods Fluids, 91 (2019), pp. 533-556.

[29] L. P. Franca And E. Dutra do Carmo, The Galerkin gradient lest-squares method, Comput. Methods Apply. Mech. Engrg., 74 (1989), pp. 41-54.

[30] L. P. Franca And S. L. Frey, Stabilized finite element methods. ii. the incompressible NavierStokes equations, Comput. Methods Appl. Mech. Engrg., 99 (1992), pp. 209-233.

[31] L. P. Franca And T. J. Hughes, Two classes of mixed finite element methods, Comput. Methods Appl. Mech. Engrg., 69 (1988), pp. 89-129.

[32] G. Fu, J. GuZmán, And M. NeILAn, Exact smooth piecewise polynomial sequences on Alfeld splits, Math. Comp., 89 (2020), pp. 1059-1091.

[33] N. R. GaUger, A. Linke, AND P. W. SChroeder, On high-order pressure-robust space discretisations, their advantages for incompressible high Reynolds number generalised Beltrami flows and beyond, SMAI J. Comput. Math., 5 (2019), pp. 89-129.

[34] V. Girault and P.-A. Raviart, Finite Element Methods for Navier-Stokes Equations: Theory and Algorithms, Springer Ser. Comp. Math. 5, Springer, New York, 1986.

[35] J. Gopalakrishnan, P. L. Lederer, And J. SchöBerl, A mass conserving mixed stress formulation for Stokes flow with weakly imposed stress symmetry, SIAM J. Numer. Anal., 58 (2020), pp. 706-732.

[36] J. Guzmán, A. Lischke, And M. Neilan, Exact sequences on Powell-Sabin splits, Calcolo, 57 (2020), pp. 1-25.

[37] J. Guzmán And M. Neilan, Conforming and divergence-free Stokes elements in three dimensions, IMA J. Numer. Anal., 34 (2014), pp. 1489-1508.

[38] J. Guzmán and M. Neilan, Conforming and divergence-free Stokes elements on general triangular meshes, Math. Comput., 83 (2014), pp. 15-36.

[39] J. GuZmán and M. NeILAn, Inf-sup stable finite elements on barycentric refinements producing divergence-free approximations in arbitrary dimensions, SIAM J. Numer. Anal., 56 (2018), pp. 2826-2844, https://doi.org/10.1137/17M1153467.

[40] J. Guzmán And L. R. Scott, The Scott-Vogelius finite elements revisited, Math. Comp., 88 (2019), pp. 515-529, https://doi.org/10.1090/mcom/3346.

[41] V. John, Finite Element Methods for Incompressible Flow Problems, Springer Ser. Comput. Math. 51, Springer, New York, 2016.

[42] V. John, A. Linke, C. Merdon, M. Neilan, and L. G. Rebholz, On the divergence constraint in mixed finite element methods for incompressible flows, SIAM Rev., 59 (2017), pp. $492-544$.

Copyright (c) by SIAM. Unauthorized reproduction of this article is prohibited. 
[43] G. Kanschat and N. Sharma, Divergence-conforming discontinuous Galerkin methods and $c^{0}$ interior penalty methods, SIAM J. Numer. Anal., 52 (2014), pp. 1822-1842, https: //doi.org/10.1137/120902975.

[44] K. L. A. KIRK AND S. Rhebergen, Analysis of a pressure-robust hybridized discontinuous Galerkin method for the stationary Navier-Stokes equations, J. Sci. Comput., 81 (2019), pp. 881-897.

[45] P. L. Lederer, C. Lehrenfeld, And J. Schöberl, Hybrid discontinuous Galerkin methods with relaxed $H$ (div)-conformity for incompressible flows. Part I, SIAM J. Numer. Anal., 56 (2018), pp. 2070-2094.

[46] P. L. Lederer, C. MERdon, AND J. SChÖBERL, Refined a posteriori error estimation for classical and pressure-robust Stokes finite element methods, Numer. Math., 142 (2019), pp. $713-748$.

[47] A. Linke, G. Matthies, And L. Tobiska, Robust arbitrary order mixed finite element methods for the incompressible Stokes equations with pressure independent velocity errors, ESAIM Math. Model. Numer. Anal., 50 (2016), pp. 289-309.

[48] A. Linke And C. Merdon, Pressure-robustness and discrete Helmholtz projectors in mixed finite element methods for the incompressible Navier-Stokes equations, Comput. Methods Appl. Mech. Engrg., 311 (2016), pp. 304-326.

[49] A. Linke And L. G. Rebholz, Pressure-induced locking in mixed methods for time-dependent (Navier-)Stokes equations, J. Comput. Phys., 388 (2019), pp. 350-356.

[50] A. LogG, K.-A. Mardal, and G. N. Wells, Automated Solution of Differential Equations by the Finite Element Method, Springer, 2012, https://doi.org/10.1007/978-3-642-23099-8.

[51] G. Matthies And L. Tobiska, Local projection type stabilization applied to inf-sup stable discretizations of the Oseen problem, IMA J. Numer. Anal., 35 (2015), pp. 239-269.

[52] A. Natale And C. J. CotTer, Scale-selective dissipation in energy-conserving finite-element schemes for two-dimensional turbulence, Quart. J. Roy. Meteorological Soc., 143 (2017), pp. $1734-1745$.

[53] M. NeIlan, Discrete and conforming smooth de Rham complexes in three dimensions, Math. Comp., 84 (2015), pp. 2059-2081.

[54] J. QIn, On the Convergence of Some Low Order Mixed Finite Elements for Incompressible Fluids, Ph.D. thesis, Pennsylvania State University, 1994.

[55] S. Rhebergen and G. N. Wells, An embedded-hybridized discontinuous Galerkin finite element method for the Stokes equations, Comput. Methods Appl. Mech. Engrg., 358 (2020), pp. 112619, 18 .

[56] P. W. Schroeder, C. Lehrenfeld, A. Linke, And G. Lube, Towards computable flows and robust estimates for inf-sup stable FEM applied to the time-dependent incompressible Navier-Stokes equations, SeMA J., 75 (2018), pp. 629-653, https://doi.org/10.1007/ s40324-018-0157-1.

[57] P. W. Schroeder And G. Lube, Pressure-robust analysis of divergence-free and conforming FEM for evolutionary incompressible Navier-Stokes flows, J. Numer. Math., 25 (2017), pp. 249-276.

[58] P. W. Schroeder And G. Lube, Divergence-free H(div)-FEM for time-dependent incompressible flows with applications to high Reynolds number vortex dynamics, J. Sci. Comput., 75 (2018), pp. 830-858.

[59] L. R. Scott And M. Vogelius, Norm estimates for a maximal right inverse of the divergence operator in spaces of piecewise polynomials, RAIRO Modél. Math. Anal. Numér., 19 (1985), pp. 111-143, https://doi.org/10.1051/m2an/1985190101111.

[60] L. ToBiska, Analysis of a new stabilized higher order finite element method for advectiondiffusion equations, Comput. Methods Appl. Mech. Engrg., 196 (2006), pp. 538-550.

[61] R. Verfürth AND P. ZANOtTi, A quasi-optimal Crouzeix-Raviart discretization of the Stokes equations, SIAM J. Numer. Anal., 57 (2019), pp. 1082-1099.

[62] U. Wilbrandt, C. Bartsch, N. Ahmed, N. Alia, F. Anker, L. Blank, A. Caiazzo, S. Ganesan, S. Giere, G. Matthies, R. Meesala, A. Shamim, J. Venkatesan, and V. John, ParMooN-A modernized program package based on mapped finite elements, Comput. Math. Appl., 74 (2017), pp. 74-88, https://doi.org/10.1016/j.camwa.2016.12.020.

[63] S. Zhang, A new family of stable mixed finite elements for the $3 D$ Stokes equations, Math. Comp., 74 (2005), pp. 543-554.

Copyright (C) by SIAM. Unauthorized reproduction of this article is prohibited. 